\title{
ON THE NUMBER OF FULLY PACKED LOOP CONFIGURATIONS WITH A FIXED ASSOCIATED MATCHING
}

\author{
F. Caselli*\#, C. Krattenthaler ${ }^{*}$, B. Lass* and P. Nadeau ${ }^{\dagger}$ \\ *Institut Camille Jordan, Université Claude Bernard Lyon-I, \\ 21, avenue Claude Bernard, F-69622 Villeurbanne Cedex, France. \\ E-mail: (caselli,kratt, lass) @euler.univ-lyon1.fr \\ ${ }^{\dagger}$ Laboratoire de Recherche en Informatique, Université Paris-Sud \\ 91405 Orsay Cedex, France \\ E-mail: nadeau@lri.fr
}

Submitted: Feb 17, 2005; Accepted: Mar 14, 2005; Published: Apr 6, 2005

Dedicated to Richard Stanley

\begin{abstract}
We show that the number of fully packed loop configurations corresponding to a matching with $m$ nested arches is polynomial in $m$ if $m$ is large enough, thus essentially proving two conjectures by Zuber [Electronic J. Combin. 11(1) (2004), Article \#R13].
\end{abstract}

\section{INTRODUCTION}

In this paper we continue the enumerative study of fully packed loop configurations corresponding to a prescribed matching begun by the first two authors in [2], where we proved two conjectures by Zuber [22] on this subject matter. (See also [6, 7, 8, 9] for related results.) The interest in this study originates in conjectures by Razumov and Stroganov [18], and by Mitra, Nienhuis, de Gier and Batchelor [17], which predict that the coordinates of the groundstate vectors of certain Hamiltonians in the dense $O(1)$ loop model are given by the number of fully packed loop configurations corresponding to particular matchings. Another motivation comes from the well-known fact (see e.g. [6, Sec. 3]) that fully packed loop configurations are in bijection with configurations in the

2000 Mathematics Subject Classification. Primary 05A15; Secondary 05B45 05E05 05E10 82B23.

Key words and phrases. Fully packed loop model, rhombus tilings, hook-content formula, nonintersecting lattice paths.

* Research supported by EC's IHRP Programme, grant HPRN-CT-2001-00272, "Algebraic Combinatorics in Europe". The second author was also partially supported by the "Algebraic Combinatorics" Programme during Spring 2005 of the Institut Mittag-Leffler of the Royal Swedish Academy of Sciences.

\#Current address: Dipartimento di Matematica, Università di Roma La Sapienza, P.le A. Moro 3, I-00185 Roma, Italy. 
six vertex model, which, in their turn, are in bijection with alternating sign matrices, and, thus, the enumeration of fully packed loop configurations corresponding to a prescribed matching constitutes an interesting refinement of the enumeration of configurations in the six vertex model or of alternating sign matrices.

Here we consider configurations with a growing number of nested arches. We show that the number of configurations is polynomial in the number of nested arches, thus proving two further conjectures of Zuber from [22].

In order to explain these conjectures, we have to briefly recall the relevant definitions from [2, 22]. The fully packed loop model (FPL model, for short; see for example [1]) is a model of (not necessarily closed) polygons on a lattice such that each vertex of the lattice is on exactly one polygon. Whether or not these polygons are closed, we will refer to them as loops.

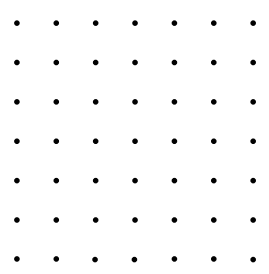

Figure 1.1. The square grid $Q_{7}$

Throughout this article, we consider this model on the square grid of side length $n-1$, which we denote by $Q_{n}$. See Figure 1.1 for a picture of $Q_{7}$. The polygons consist of horizontal or vertical edges connecting vertices of $Q_{n}$, and edges that lead outside of $Q_{n}$ from a vertex along the border of $Q_{n}$, see Figure 1.2 for an example of an allowed configuration in the FPL model. We call the edges that stick outside of $Q_{n}$ external links. The reader is referred to Figure 2.1 for an illustration of the external links of the square $Q_{11}$. (The labels should be ignored at this point.) It should be noted that the four corner points are incident to a horizontal and a vertical external link. We shall be interested here in allowed configurations in the FPL model, in the sequel referred to as FPL configurations, with periodic boundary conditions. These are FPL configurations where, around the border of $Q_{n}$, every other external link of $Q_{n}$ is part of a polygon. The FPL configuration in Figure 1.2 is in fact a configuration with periodic boundary conditions.

Every FPL configuration defines in a natural way a (non-crossing) matching of the external links by matching those which are on the same polygon (loop). We are interested in the number of FPL configurations corresponding to a fixed matching. Thanks to a theorem of Wieland [21] (see Theorem 2.1), this number is invariant if the matching is rotated around $Q_{n}$. This allows one to represent a matching in form of a chord diagram of $2 n$ points placed around a circle, see Figure 1.3 for the chord diagram representation of the matching corresponding to the FPL configuration in Figure 1.2. 


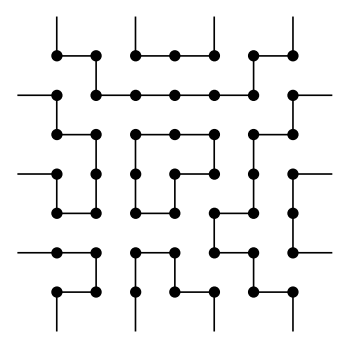

Figure 1.2. An FPL configuration on $Q_{7}$ with periodic boundary conditions

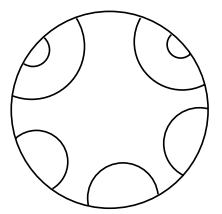

FIGURE 1.3. The chord diagram representation of a matching

The two conjectures by Zuber which we address in this paper concern FPL configurations corresponding to a matching with $m$ nested arches. More precisely, let $X$ represent a fixed (non-crossing) matching with $n-m$ arches. By adding $m$ nested arches, we obtain a certain matching. (See Figure 1.4 for a schematic picture of the matching which is composed in this way.) The first of Zuber's conjectures states that the number of FPL configurations which has this matching as associated matching is polynomial in $\mathrm{m}$. In fact, the complete statement is even more precise. It makes use of the fact that to any matching $X$ one can associate a Ferrers diagram $\lambda(X)$ in a natural way (see Section 2.4 for a detailed explanation).

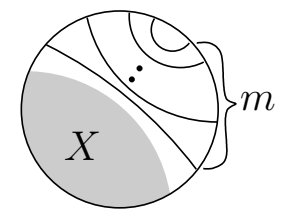

Figure 1.4. The matching composed out of a matching $X$ and $m$ nested arches

Conjecture 1.1 ([22, Conj. 6]). Let $X$ be a given non-crossing matching with $n-m$ arches, and let $X \cup m$ denote the matching arising from $X$ by adding $m$ nested arches. Then the number $A_{X}(m)$ of FPL configurations which have $X \cup m$ as associated matching is equal to $\frac{1}{|X| !} P_{X}(m)$, where $P_{X}(m)$ is a polynomial of degree $|\lambda(X)|$ with integer coefficients, and its highest degree coefficient is equal to $\operatorname{dim}(\lambda(X))$. Here, $|\lambda(X)|$ denotes the size of the Ferrers diagram $\lambda(X)$, and $\operatorname{dim}(\lambda(X))$ denotes the dimension of the irreducible representation of the symmetric group $S_{|\lambda(X)|}$ indexed by the Ferrers diagram $\lambda(X)$ (which is given by the hook formula; see (2.1)). 
The second conjecture of Zuber generalizes Conjecture 1.1 to the case where a bundle of nested arches is squeezed between two given matchings. More precisely, let $X$ and $Y$ be two given (non-crossing) matchings. We produce a new matching by placing $X$ and $Y$ along our circle that we use for representing matchings, together with $m$ nested arches which we place in between. (See Figure 1.5 for a schematic picture.) We denote this matching by $X \cup m \cup Y$.

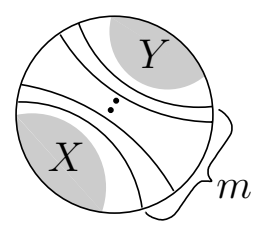

Figure 1.5. Squeezing $m$ nested arches between two matchings $X$ and $Y$

Conjecture 1.2 ([22, Conj. 7]). Let $X$ and $Y$ be two non-crossing matchings. Then the number $A_{X, Y}(m)$ of FPL configurations which have $X \cup m \cup Y$ as associated matching is equal to $\frac{1}{|\lambda(X)| !|\lambda(Y)| !} P_{X, Y}(m)$, where $P_{X, Y}(m)$ is a polynomial of degree $|\lambda(X)|+|\lambda(Y)|$ with integer coefficients, and its highest degree coefficient is equal to $\operatorname{dim}(\lambda(X)) \cdot \operatorname{dim}(\lambda(Y))$.

It is clear that Conjecture 1.2 is a generalization of Conjecture 1.1 , since $A_{X}(m)=$ $A_{X, \emptyset}(m)$ for any non-crossing matching $X$, where $\emptyset$ denotes the empty matching. Nevertheless, we shall treat both conjectures separately, because this will allow us to obtain, in fact, sharper results than just the statements in the conjectures, with our result covering Conjecture 1.1 - see Theorem 4.2 and Section 5 - being more precise than the corresponding result concerning Conjecture 1.2 - see Theorem 6.7. We must stress at this point that, while we succeed to prove Conjecture 1.1 completely, we are able to prove Conjecture 1.2 only for "large" $m$, see the end of Section 6 for the precise statement. There we also give an explanation of the difficulty of closing the gap.

We conclude the introduction by outlining the proofs of our results, and by explaining the organisation of our paper at the same time. All notation and prerequisites that we are going to use in these proofs are summarized in Section 2 below.

Our proofs are based on two observations due to de Gier in [6, Sec. 3] (as are the proofs in $[2,7,9])$ : if one considers the FPL configurations corresponding to a given matching which has a big number of nested arches, there are many edges which are occupied by any such FPL configuration. We explain this observation, with focus on our particular problem, in Section 3. As a consequence, we can split our enumeration problem into the problem of enumerating configurations in two separate subregions of $Q_{n}$, see the explanations accompanying Figure 4.1, respectively Figure 6.2. While one of the regions does not depend on $m$, the others grow with $m$. It remains the task of establishing that the number of configurations in the latter subregions grows polynomially with $m$. In order to do so, we use the second observation of de Gier, namely the existence of a bijection between FPL configurations (subject to certain constraints on the edges) and rhombus 
tilings, see the proofs of Theorem 4.2 and Lemma 6.4. In the case of Conjecture 1.1, the rhombus tilings can be enumerated by an application of the hook-content formula (recalled in Theorem 2.2), while in the case of Conjecture 1.2 we use a standard correspondence between rhombus tilings and non-intersecting lattice paths, followed by an application of the Lindström-Gessel-Viennot theorem (recalled in Lemma 2.3), to obtain a determinant for the number of rhombus tilings, see the proof of Lemma 6.4. In both cases, the polynomial nature of the number of rhombus tilings is immediately obvious, if $m$ is "large enough." To cover the case of "small" $m$ of Conjecture 1.1 as well, we employ a somewhat indirect argument, which is based on a variation of the above reasoning, see Section 5. Finally, for the proof of the more specific assertions in Conjectures 1.1 and 1.2 on the integrality of the coefficients of the polynomials (after renormalization) and on the leading coefficient, we need several technical lemmas (to be precise, Lemmas 4.1, 6.2 and 6.6). These are implied by Theorem 7.1 (see also Corollary 7.4), which is the subject of Section 7.

\section{Preliminaries}

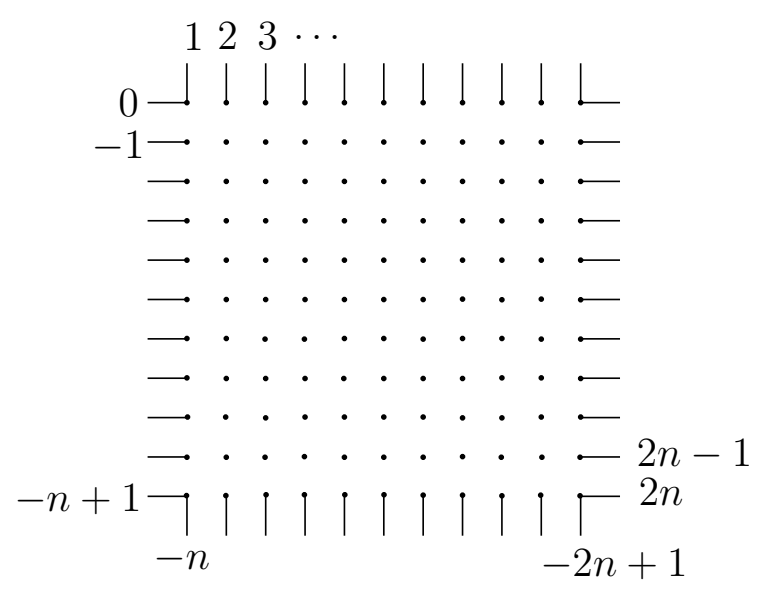

FiguRE 2.1. The labelling of the external links

2.1. Notation and conventions concerning FPL configurations. The reader should recall from the introduction that any FPL configuration defines a matching on the external links occupied by the polygons, by matching those which are on the same polygon. We call this matching the matching associated to the FPL configuration. When we think of the matching as being fixed, and when we consider all FPL configurations having this matching as associated matching, we shall also speak of these FPL configurations as the "FPL configurations corresponding to this fixed matching."

We label the $4 n$ external links around $Q_{n}$ by $\{-2 n+1,-2 n+2, \ldots, 2 n-1,2 n\}$ clockwise starting from the right-most link on the bottom side of the square, see Figure 2.1. If $\alpha$ is an external link of the square, we denote its label by $L(\alpha)$. Throughout this paper, all the 
FPL configurations that are considered are configurations which correspond to matchings of either the even labelled external links or the odd labelled external links.

2.2. Wieland's rotational invariance. Let $X$ be a non-crossing matching of the set of even (odd) labelled external links. Let $\tilde{X}$ be the "rotated" matching of the odd (even) external links defined by the property that the links labelled $i$ and $j$ in $X$ are matched if and only if the links labelled $i+1$ and $j+1$ are matched in $\tilde{X}$, where we identify $2 n+1$ and $-2 n+1$. Let $F P L(X)$ denote the set of FPL configurations corresponding to the matching $X$. Wieland [21] proved the following surprising result.

Theorem 2.1 (Wieland). For any matching $X$ of the even (odd) labelled external links, we have

$$
|F P L(X)|=|F P L(\tilde{X})| .
$$

In other terms, the number of FPL configurations corresponding to a given matching is invariant under rotation of the "positioning" of the matching around the square. As we mentioned already in the introduction, this being the case, we can represent matchings in terms of chord diagrams of $2 n$ points placed around a circle (see Figure 1.3).

2.3. Partitions and Ferrers diagrams. Next we explain our notation concerning partitions and Ferrers diagrams (see e.g. [20, Ch. 7]). A partition is a vector $\lambda=\left(\lambda_{1}, \lambda_{2}, \ldots, \lambda_{\ell}\right)$ of positive integers such that $\lambda_{1} \geq \lambda_{2} \geq \cdots \geq \lambda_{\ell}$. For convenience, we shall sometimes use exponential notation. For example, the partition $(3,3,3,2,1,1)$ will also be denoted as $\left(3^{3}, 2,1^{2}\right)$. To each partition $\lambda$, one associates its Ferrers diagram, which is the leftjustified arrangement of cells with $\lambda_{i}$ cells in the $i$-th row, $i=1,2, \ldots, \ell$. See Figure 2.3 for the Ferrers diagram of the partition $(7,5,2,2,1,1)$. (At this point, the labels should be disregarded.) We will usually identify a Ferrers diagram with the corresponding partition; for example we will say "the Ferrers diagram $\left(\lambda_{1}, \ldots, \lambda_{\ell}\right)$ " to mean "the Ferrers diagram corresponding to the partition $\left(\lambda_{1}, \ldots, \lambda_{\ell}\right)$ ". The size $|\lambda|$ of a Ferrers diagram $\lambda$ is given by the total number of cells of $\lambda$. The partition conjugate to $\lambda$ is the partition $\lambda^{\prime}=\left(\lambda_{1}^{\prime}, \lambda_{2}^{\prime}, \ldots, \lambda_{\lambda_{1}}^{\prime}\right)$, where $\lambda_{j}^{\prime}$ is the length of the $j$-th column of the Ferrers diagram of $\lambda$.

Given a Ferrers diagram $\lambda$, we write $(i, j)$ for the cell in the $i$-th row and the $j$-th column of $\lambda$. We use the notation $u=(i, j) \in \lambda$ to express the fact that $u$ is a cell of $\lambda$. Given a cell $u$, we denote by $c(u):=j-i$ the content of $u$ and by $h(u):=\lambda_{i}+\lambda_{j}^{\prime}-i-j+1$ the hook length of $u$, where $\lambda$ is the partition associated to $\lambda$.

It is well-known (see e.g. [11, p. 50]), that the dimension of the irreducible representation of the symmetric group $S_{|\lambda|}$ indexed by a partition (or, equivalently, by a Ferrers diagram) $\lambda$, which we denote by $\operatorname{dim}(\lambda)$, is given by the hook-length formula due to Frame, Robinson and Thrall [10],

$$
\operatorname{dim}(\lambda)=\frac{n !}{\prod_{u \in \lambda} h_{u}}
$$


2.4. How to associate a Ferrers diagram to a matching. Let $X$ be a non-crossing matching on the set $\{1,2, \ldots, 2 d\}$, that is, an involution of this set with no fixed points which can be represented by non-crossing arches in the upper half-plane (see Figure 2.2 for an example of a non-crossing matching of the set $\{1,2, \ldots, 16\}$ ). Such a non-crossing matching can be translated into a 0-1-sequence $v(X)=v_{1} v_{2} \ldots v_{2 d}$ of length $2 d$ by letting $v_{i}=0$ if $X(i)>i$, and $v_{i}=1$ if $X(i)<i$. For example, if $X$ is the matching appearing in Figure 2.2, then $v=0010010011101101$.

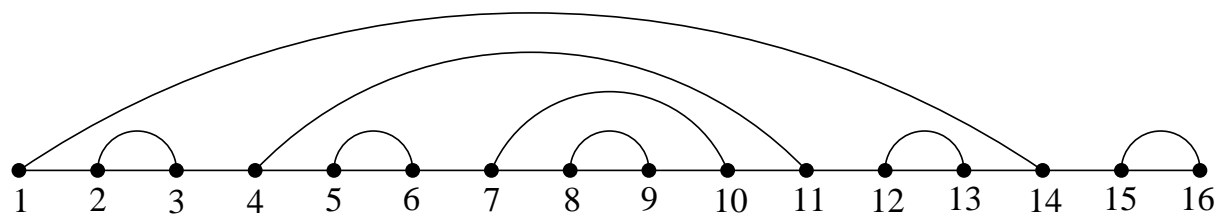

FiguRE 2.2. A planar matching

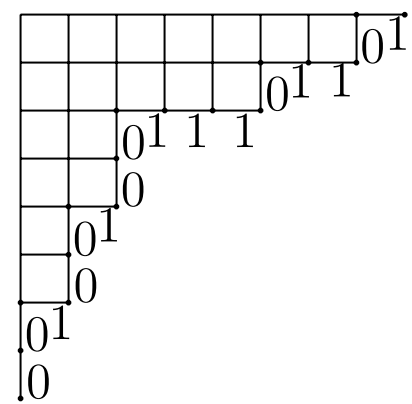

Figure 2.3. A Ferrers diagram and its $d$-code

On the other hand, any 0-1-sequence can be translated into a Ferrers diagram by reading the 0-1-sequence from left to right and interpreting a 0 as a unit up-step and a 1 as unit right-step. From the starting point of the obtained path we draw a vertical segment upwards, and from the end point a horizontal segment left-wards. By definition, the region enclosed by the path, the vertical and the horizontal segment is the Ferrers diagram associated to the given matching. See Figure 2.3 for the Ferrers diagram associated to the matching in Figure 2.2. (In the figure, for the sake of clarity, we have labelled the up-steps of the path by 0 and its right-steps by 1.) In the sequel, we shall denote the Ferrers diagram associated to $X$ by $\lambda(X)$.

Conversely, given a Ferrers diagram $\lambda$, there are several 0 -1-sequences which produce $\lambda$ by the above described procedure. Namely, by moving along the lower/right boundary of $\lambda$ from lower-left to top-right, and recording a 0 for every up-step and a 1 for every right-step, we obtain one such 0-1-sequence. Prepending an arbitrary number of 0's and appending an arbitrary number of 1's we obtain all the other sequences which give rise to $\lambda$ by the above procedure. Out of those, we shall make particular use of the so-called 
$d$-code of $\lambda$ (see [20, Ex. 7.59]). Here, $d$ is a positive integer such that $\lambda$ is contained in the Ferrers diagram $\left(d^{d}\right)$. We embed $\lambda$ in $\left(d^{d}\right)$ so that the diagram $\lambda$ is located in the top-left corner of the square $\left(d^{d}\right)$. We delete the lower side and the right side of the square $\left(d^{d}\right)$. (See Figure 2.3 for an example where $d=8$ and $\lambda=(7,5,2,2,1,1)$.) Now, starting from the lower/left corner of the square, we move, as before, along the lower/right boundary of the figure from lower-left to top-right, recording a 0 for every up-step and a 1 for every right-step. By definition, the obtained 0-1-sequence is the $d$-code of $\lambda$. Clearly, the $d$-code has exactly $d$ occurrences of 0 and $d$ occurrences of 1 . For example, the 8-code of the Ferrers diagram $(7,5,2,2,1,1)$ is 0010010011101101.

2.5. An enumeration result for rhombus tilings. In the proof of Theorem 4.2, we shall need a general result on the enumeration of rhombus tilings of certain subregions of the regular triangular lattice in the plane, which are indexed by Ferrers diagrams. (Here, and in the sequel, by a rhombus tiling we mean a tiling by rhombi of unit side lengths and angles of $60^{\circ}$ and $120^{\circ}$.) This result appeared in an equivalent form in [2, Theorem 2.6]. As is shown there, it follows from Stanley's hook-content formula [19, Theorem 15.3], via the standard bijection between rhombus tilings and non-intersecting lattice paths, followed by the standard bijection between non-intersecting lattice paths and semistandard tableaux.

Let $\lambda$ be a Ferrers diagram contained in the square $\left(d^{d}\right)$, and let $h$ be a non-negative integer $h$. We define the region $R(\lambda, d, h)$ to be a pentagon with some notches along the top side. More precisely (see Figure 2.4 where the region $R(\lambda, 8,3)$ is shown, with $\lambda$ the Ferrers diagram $(7,5,2,2,1,1)$ from Figure 2.3), the region $R(\lambda, d, h)$ is the pentagon with base side and bottom-left side equal to $d$, top-left side $h$, a top side of length $2 d$ with notches which will be explained in just a moment, and right side equal to $d+h$. To determine the notches along the top side, we read the $d$-code of $\lambda$, and we put a notch whenever we read a 0 , while we leave a horizontal piece whenever we read a 1 .

\section{$d$-code of $\lambda$}

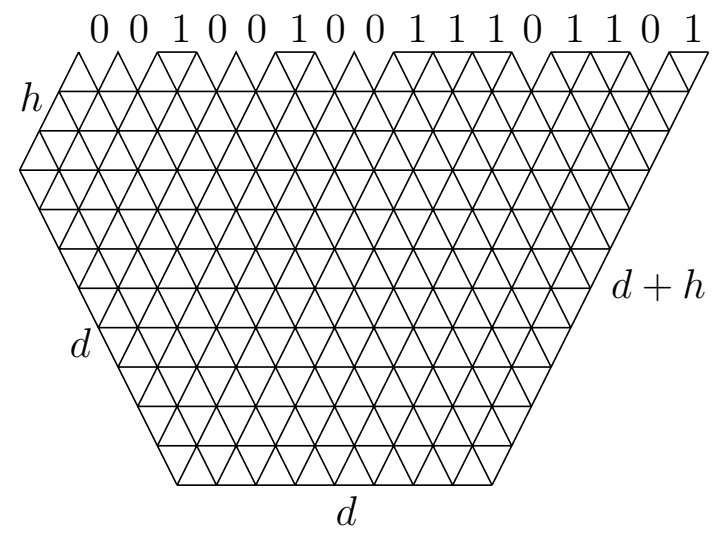

Figure 2.4. The region $R(\lambda, d, h)$

We can now state the announced enumeration result for rhombus tilings of the regions $R(\lambda, d, h)$. 
Theorem 2.2. Given a Ferrers diagram $\lambda$ contained in the square $\left(d^{d}\right)$ and a positive integer $h$, the number of rhombus tilings of the region $R(\lambda, d, h)$ is given by $S S Y T(\lambda, d+h)$, where

$$
\operatorname{SSYT}(\lambda, N)=\prod_{u \in \lambda} \frac{c(u)+N}{h(u)},
$$

with $c(u)$ and $h(u)$ the content and the hook length of u, respectively, as defined in Section 2.3.

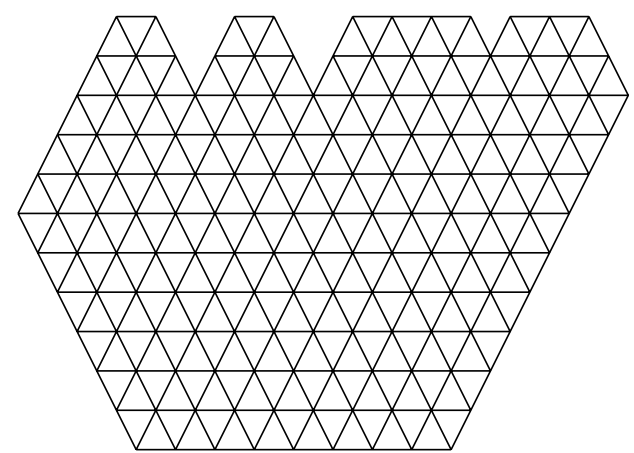

FiguRE 2.5. The reduced region

Remark. The choice of the notation $\operatorname{SSYT}(\lambda, N)$ comes from the fact that the number in (2.2) counts at the same time the number of semistandard tableaux of shape $\lambda$ with entries at most $N$ (cf. [19, Theorem 15.3]). Indeed, implicitly in the proof of the above theorem which we give below is a bijection between the rhombus tilings in the statement of the theorem and these semistandard tableaux.

Proof of Theorem 2.2. It should be observed that, due to the nature of the region $R(\lambda, d, h)$, there are several "forced" subregions, that is, subregions where the tiling is completely determined. For example, the right-most layer in Figure 2.4 must necessarily be completely filled with right-oriented rhombi, while the first two upper-left layers must be filled with horizontally symmetric rhombi. If we remove all the "forced" rhombi, then a smaller region remains. See Figure 2.5 for the result of this reduction applied to the region in Figure 2.4. To the obtained region we may apply Theorems 2.6 and 2.5 from [2]. As a result, we obtain the desired formula.

2.6. The Lindström-Gessel-Viennot formula. It is well-known that rhombus tilings are (usually) in bijection with families of non-intersecting lattice paths. We shall make use of this bijection in Section 6, together with the main result on the enumeration of non-intersecting lattice paths, which is a determinantal formula due to Lindström [16]. In the combinatorial literature, it is most often attributed to Gessel and Viennot [12, 13], but it can actually be traced back to Karlin and McGregor [14, 15]. 


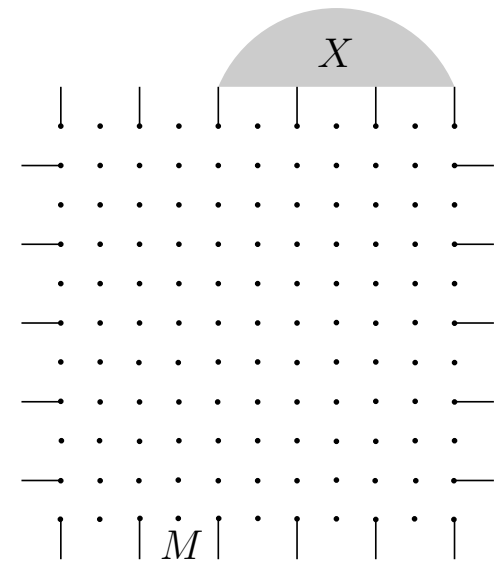

FiguRE 3.1. Placing the matching around $Q_{n}$

Let us briefly recall that formula, or, more precisely, a simplified version tailored for our purposes. We consider lattice paths in the planar integer lattice $\mathbb{Z}^{2}$ consisting of unit horizontal and vertical steps in the positive direction. Given two points $A$ and $E$ in $\mathbb{Z}^{2}$, we write $\mathcal{P}(A \rightarrow E)$ for the number of paths starting at $A$ and ending at $E$. We say that a family of paths is non-intersecting if no two paths in the family have a point in common.

We can now state the announced main result on non-intersecting lattice paths (see [16, Lemma 1] or [13, Cor. 2]).

Lemma 2.3. Let $A_{1}, A_{2}, \ldots, A_{n}, E_{1}, E_{2}, \ldots, E_{n}$ be points of the planar integer lattice $\mathbb{Z}^{2}$, such that for all $i<j$ the point $A_{i}$ is (weakly) south-east of the point $A_{j}$, and the point $E_{i}$ is (weakly) south-east of the point $E_{j}$. Then the number of families $\left(P_{1}, P_{2}, \ldots, P_{n}\right)$ of non-intersecting lattice paths, $P_{i}$ running from $A_{i}$ to $E_{i}, i=1,2, \ldots, n$, is given by

$$
\operatorname{det}\left(\mathcal{P}\left(A_{i} \rightarrow E_{j}\right)\right)_{1 \leq i, j \leq n} \text {. }
$$

\section{FIXED EDGES}

In this section, we perform the first step in order to prove Conjecture 1.1. Let $X$ be a given non-crossing matching with $d$ arches. As in the statement of the conjecture, let $X \cup m$ be the matching obtained by adding $m$ nested arches to $X$. Thanks to Theorem 2.1 of Wieland, we may place $X \cup m$ in an arbitrary way around $Q_{n}=Q_{d+m}$, without changing the number of corresponding FPL configurations. We place $X \cup m$ so that, using Lemma 3.1 below, the FPL configurations corresponding to the matching will have as many forced edges as possible.

To be precise, we place $X \cup m$ so that the arches corresponding to $X$ appear on the very right of the upper side of $Q_{n}$. That is, we place these arches on the external links labelled $n-4 d+2, n-4 d+4, \ldots, n-2, n$. Equivalently, we choose to place the centre $M$ 
of the $m$ nested arches on the external link labelled by $-n-2 d+1$. See Figure 3.1 for a schematic picture, and Figure 3.3 for a more elaborate one (in which the added edges in the interior of the square grid $Q_{n}$ should be ignored at this point). In order to guarantee that $X$ has place along the upper side of the square grid, we must assume that $m \geq 3 d$.

The following lemma helps to identify edges which are occupied by each FPL configuration corresponding to a given matching. It is a consequence of an iterated use of a result of de Gier (see [6, Lemma 8] or [2, Lemmas 2.2 and 2.3]). In the sequel, when we speak of "fixed edges" we always mean edges that have to be occupied by any FPL configuration under consideration.

Lemma 3.1. Let $\alpha=\alpha_{1}, \alpha_{2}, \ldots, \alpha_{k}=\beta$ be a sequence of external links, where $L\left(\alpha_{i}\right)=$ $a+2 i \bmod 4 n$, for some fixed $a$, that is, the external links $\alpha_{1}, \alpha_{2}, \ldots, \alpha_{k}$ comprise every second external link along the stretch between $\alpha$ and $\beta$ along the border of $Q_{n}$ (clockwise). Furthermore, we suppose that one of the following conditions holds:

(1) $\alpha$ and $\beta$ are both on the top side of $Q_{n}$, that is, $1 \leq L(\alpha)<L(\beta) \leq n$;

(2) $\alpha$ is on the top side and $\beta$ is on the right side of $Q_{n}$, that is, $1 \leq L(\alpha) \leq n<L(\beta)$ and $n-L(\alpha)>L(\beta)-(n+1)$;

(3) $\alpha$ is on the left side and $\beta$ is on the right side of $Q_{n}$, that is, $n<L(\beta) \leq 2 n$ and $-n<L(\alpha) \leq 0$.

For the FPL configurations for which the external links $\alpha_{1}, \alpha_{2}, \ldots, \alpha_{k}$ belong to different loops, the region of fixed edges is (essentially) triangular (see Figure 3.2 for illustrations of the region and the fixed edges in its interior; the "essentially" refers to the fact that in Cases (2) and (3) parts of the triangle are cut off). More precisely, if one places the origin $O$ of the coordinate system one unit to the left of the top-left corner of $Q_{n}$, the coordinates of the triangle are given in the following way: let $A^{\prime}$ and $B^{\prime}$ be the points on the $x$-axis with $x$-coordinates $L(\alpha)$ and $L(\beta)$, respectively, then the region of fixed edges is given by the intersection of the square $Q_{n}$ and the (rectangular isosceles) triangle having the segment $A^{\prime} B^{\prime}$ as basis.

In Cases (2) and (3), the configurations are completely fixed as "zig-zag" paths in the corner regions of $Q_{n}$ where a part of the triangle was cut off (see again Figure 3.2). More precisely, in Case (2), this region is the reflexion of the corresponding cut off part of the triangle in the right side of $Q_{n}$, and in Case (3) it is that region and also the reflexion of the corresponding cut off part on the left in the left side of $Q_{n}$.

We use this lemma to determine the set of fixed edges of the FPL configurations corresponding to the matching $X \cup m$ in Conjecture 1.1. For convenience (the reader should consult Figure 3.3 while reading the following definitions), we let $A, B, C, D, E$ be the border vertices of the external links labelled $n-4 d+3, n-1, n+2 d,-n+2 d,-n+4 d-2$, respectively, we let $J$ be the intersection point of the line connecting $D$ and $M$ and the line emanating diagonally from $B$, we let $K$ be the intersection point of the latter line emanating from $B$ and the line emanating diagonally (to the right) from $A$, and we let $L$ be the intersection point of the latter line emanating from $A$ and the line connecting $C$ 


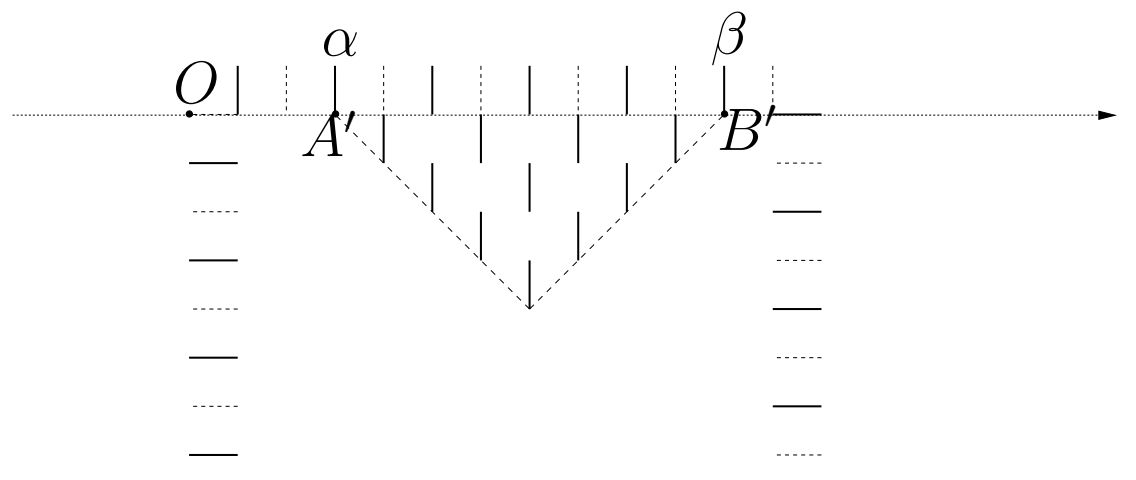

(1)

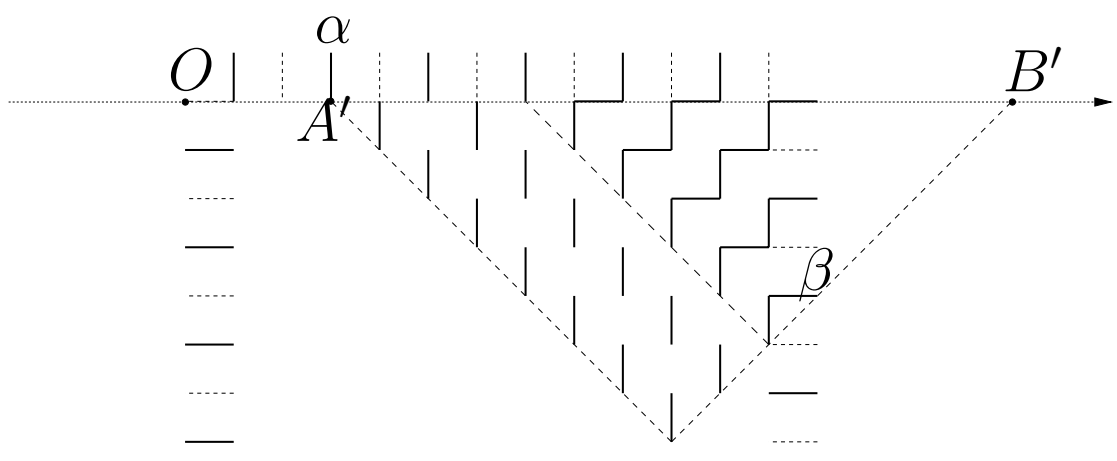

(2)

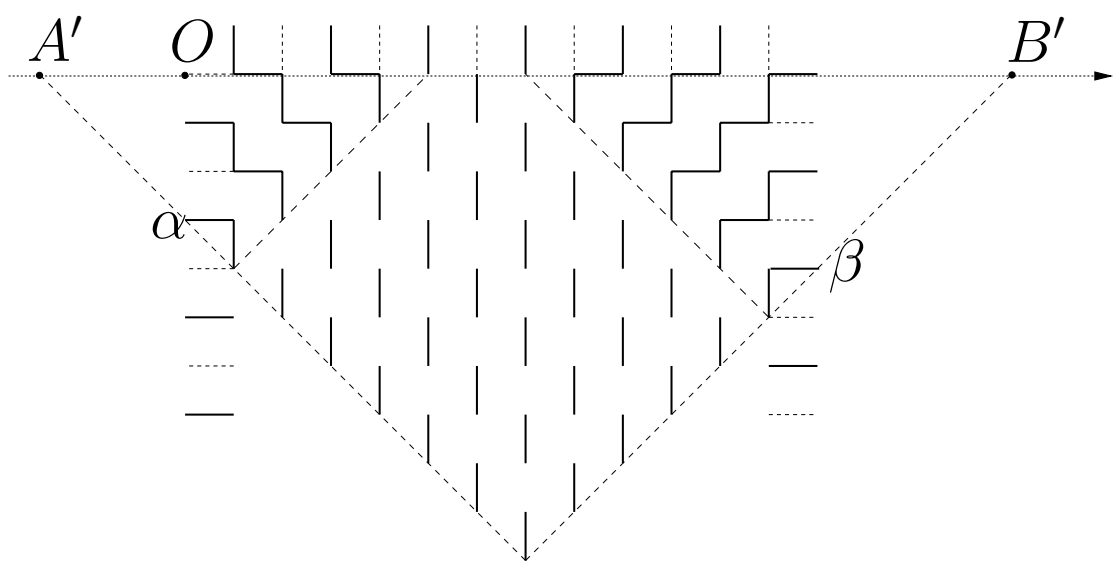

(3)

FiguRE 3.2. The possible regions of fixed edges determined by a sequence of external links belonging to distinct loops 


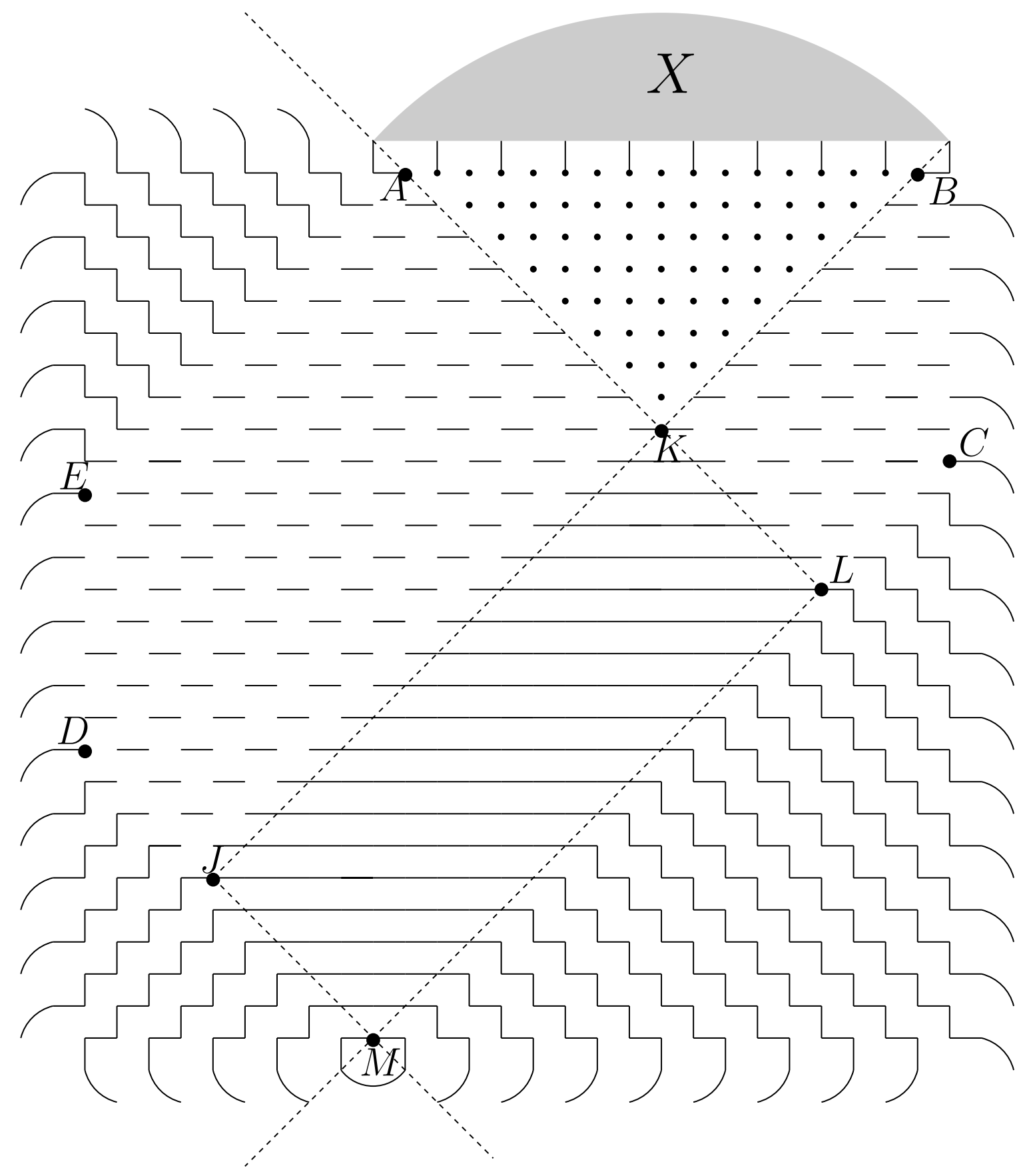

Figure 3.3. The set of fixed edges

and $M$. We state the result of the application of Lemma 3.1 to our case in form of the following lemma. 
Lemma 3.2. The region of fixed edges of the FPL configurations corresponding to the matching in Conjecture 1.1 contains all the edges indicated in Figure 3.3, that is:

(1) all the horizontal edges in the rectangular region JKLM,

(2) every other horizontal edge in the pentagonal region AKJDE as indicated in the figure,

(3) every other horizontal edge in the region BCLK as indicated in the figure,

(4) the zig-zag lines in the corner regions above the line EA, respectively below the lines $D M$ and $M C$, as indicated in the figure.

Proof. This result follows by applying Lemma 3.1 to all the external links corresponding to the $m$ nested arches which are on the left of the centre $M$ plus the "first" external link of the matching $X$, on the one hand, and to all the external links corresponding to the $m$ nested arches which are on the right of the centre $M$ plus the "last" external link of the matching $X$, on the other hand. More precisely, we apply Lemma 3.1 to the sets

$\{\alpha$ an external link with $-n-2 d+2 \leq L(\alpha) \leq n-4 d+2$ or $L(\alpha) \geq 3 n-2 d\}$

and

$$
\{\alpha \text { an external link with either } L(\alpha) \leq-n-2 d \text { or } L(\alpha) \geq n\} .
$$

The triangles forming the respective regions of fixed edges are drawn by dashed lines in Figure 3.3. Note that the two triangles of fixed edges overlap in the rectangular region $J K L M$, where the fixed edges form parallel horizontal lines.

\section{Proof of Conjecture 1.1 For $m$ LARge EnOugh}

Let $X$ be a non-crossing matching consisting of $d$ arches, and let $m$ be a positive integer such that $m \geq 3 d$. As in Conjecture 1.1, we denote the matching arising by adding $m$ nested arches to $X$ by $X \cup m$. The goal of this section is to give an explicit formula for the number $A_{X}(m)$ of FPL configurations corresponding to the matching $X \cup m$ (cf. Figure 1.4), which implies Conjecture 1.1 for $m \geq 3 d$, see Theorem 4.2.

Recall from Section 3 how we place this matching around the grid $Q_{n}=Q_{d+m}$, see Figure 3.3, where we have chosen $d=5, m=23$, and, hence, $n=d+m=28$. The reader should furthermore recall the placement of the points $A, B, C, D, E, J, K, L, M$. Let $\xi_{1}$ be the segment which connects the point which is half a unit to the left of $A$ and the point which is half a unit left of $K$, see Figure 4.1.

There are exactly $2 d-2$ possible vertical edges that cross the segment $\xi_{1}$. We denote them by $e_{1}, e_{2}, \ldots, e_{2 d-2}$, starting from the top one and proceeding south-east. We claim that among those there are exactly $d-1$ which are occupied by an FPL configuration. To see this, we first note that in the rectangular region $J K L M$ there are $m-d+1$ parallel lines strictly below $K$. Each of them must be part of one of the $m+1$ loops starting on the external links labelled $\{-n-2 d+2,-n-2 d+4, \ldots, n-4 d, n-4 d+2\}$ (these are the external links "between" $M$ and $A$, in clockwise direction). Hence there are exactly $d$ loops that cross the segment $\xi_{1}$, which implies that any FPL configuration occupies exactly $d-1$ vertical edges out of $\left\{e_{1}, e_{2}, \ldots, e_{2 d-2}\right\}$, as we claimed. We encode a choice of $d-1$ 


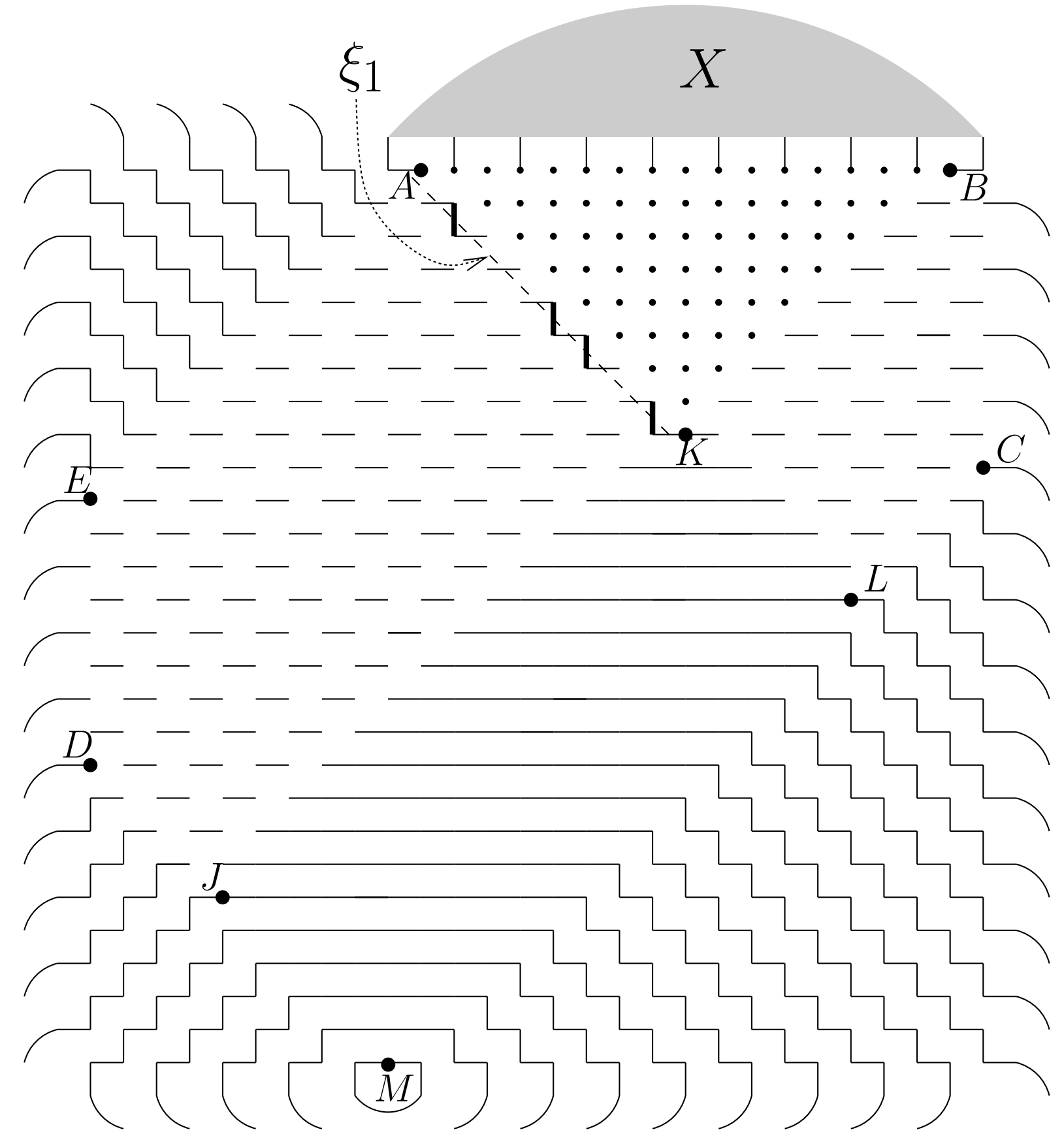

FIGURE 4.1. Splitting the problem

edges from $\left\{e_{1}, e_{2}, \ldots, e_{2 d-2}\right\}$ by a subset $\mathcal{E}$ from $\{1,2, \ldots, 2 d-2\}$, by making the obvious identification that the choice of $e_{i_{1}}, e_{i_{2}}, \ldots, e_{i_{d-1}}$ is encoded by $\mathcal{E}=\left\{i_{1}, i_{2}, \ldots, i_{d-1}\right\}$.

On the other hand, any such choice is equivalent to the choice of a Ferrers diagram contained in the square Ferrers diagram $\left((d-1)^{d-1}\right)$ by the following construction. Let THE ELECTRONiC JOURNAL OF COMBINATORICS 11(2) (2005), \#R16 


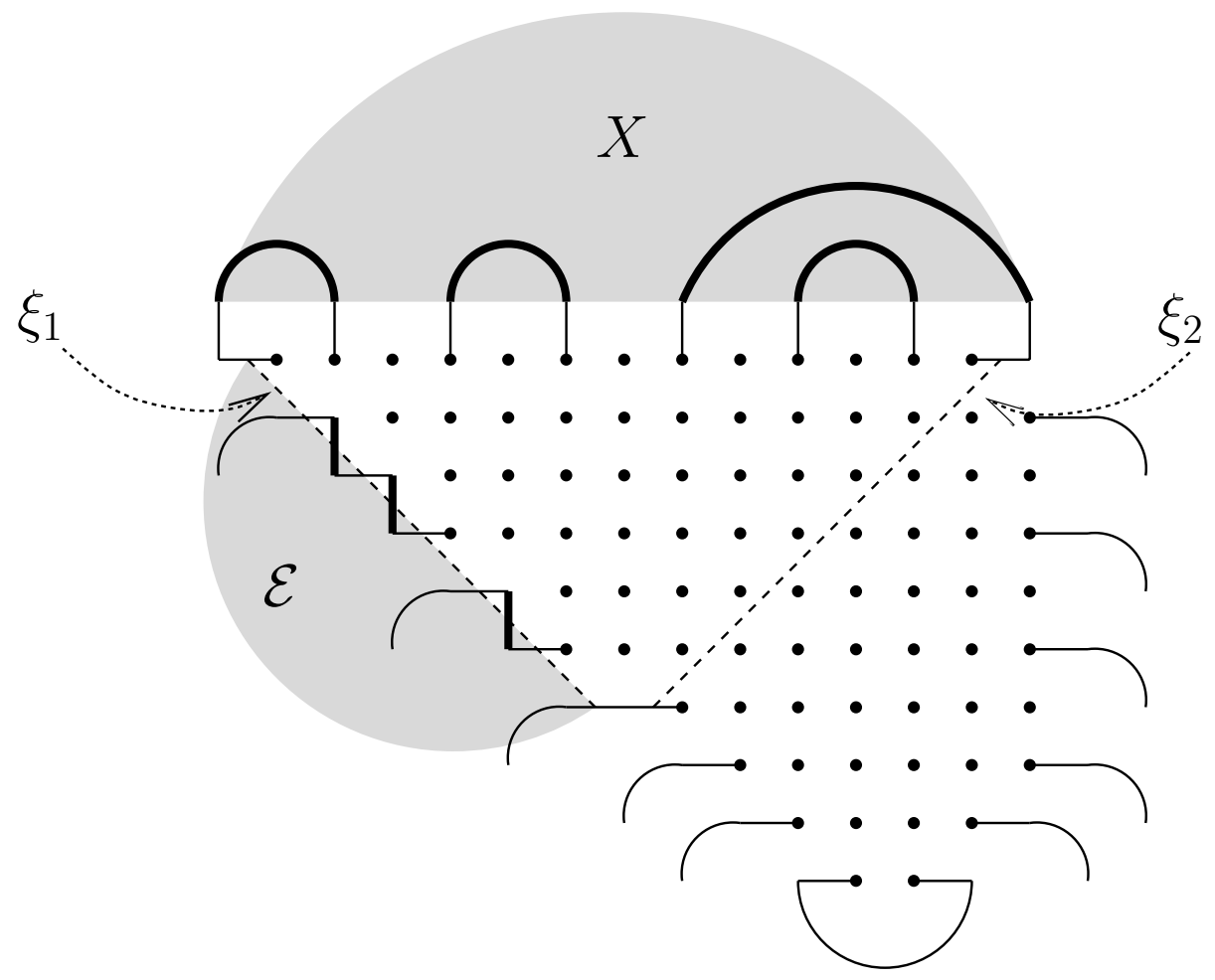

FIgURE 4.2. The numbers $a_{X}(\mathcal{E})$

$\mathcal{E} \subset\{1,2, \ldots, 2 d-2\}$ be of cardinality $d-1$. Let $c_{\mathcal{E}}:=c_{1} c_{2} \ldots c_{2 d-2}$ be the binary string defined by $c_{i}=0$ if and only if $i \in \mathcal{E}$. The string $c_{\mathcal{E}}$ obtained in this way determines a Ferrers diagram, as we described in Section 2.3. We denote this Ferrers diagram by $\lambda(\mathcal{E})$. In the example in Figure 4.1, we have $\mathcal{E}=\{2,5,6,8\}$ and, hence, $\lambda(\mathcal{E})=(4,3,3,1)$.

It is obvious from the picture, that, once we have chosen the vertical edges along $\xi_{1}$ belonging to an FPL configuration with associated matching $X \cup m$ (that is, the vertical edges out of $\left\{e_{1}, e_{2}, \ldots, e_{2 d-2}\right\}$ which are occupied by the FPL configuration), the configuration can be completed separately in the region to the "left" of $\xi_{1}$ (that is, in the region $A K J D E$ ) and to the "right" of $\xi_{1}$ (that is, in the region $A B C L K$ ). In particular, it is not difficult to see that that the number of FPL configurations with associated matching $X \cup m$ which, out of $\left\{e_{1}, e_{2}, \ldots, e_{2 d-2}\right\}$, occupy a fixed subset of vertical edges, is equal to the number of FPL configurations in the region $A K J D E$ times the number of FPL configurations in the region $A B C L K$ which respect the matching $X$.

Clearly, the region $A B C L K$ to the right of $\xi_{1}$ does not depend on $m$. We denote the number of FPL configurations of that region which respect the matching $X$ and whose set of edges from $\left\{e_{1}, e_{2}, \ldots, e_{2 d-2}\right\}$ is encoded by $\mathcal{E}$ by $a_{X}(\mathcal{E})$. For example, if $X$ is the matching $\{1 \leftrightarrow 2,3 \leftrightarrow 4,5 \leftrightarrow 6\}$, and if $\mathcal{E}$ is the set $\{1,4\}$, then we have $a_{X}(\mathcal{E})=6$. The six configurations corresponding to this choice of $X$ and $\mathcal{E}$ are shown in Figure 4.3, where the arches corresponding to $X$ and the edges corresponding to $\xi_{1}$ are marked in bold-face. 

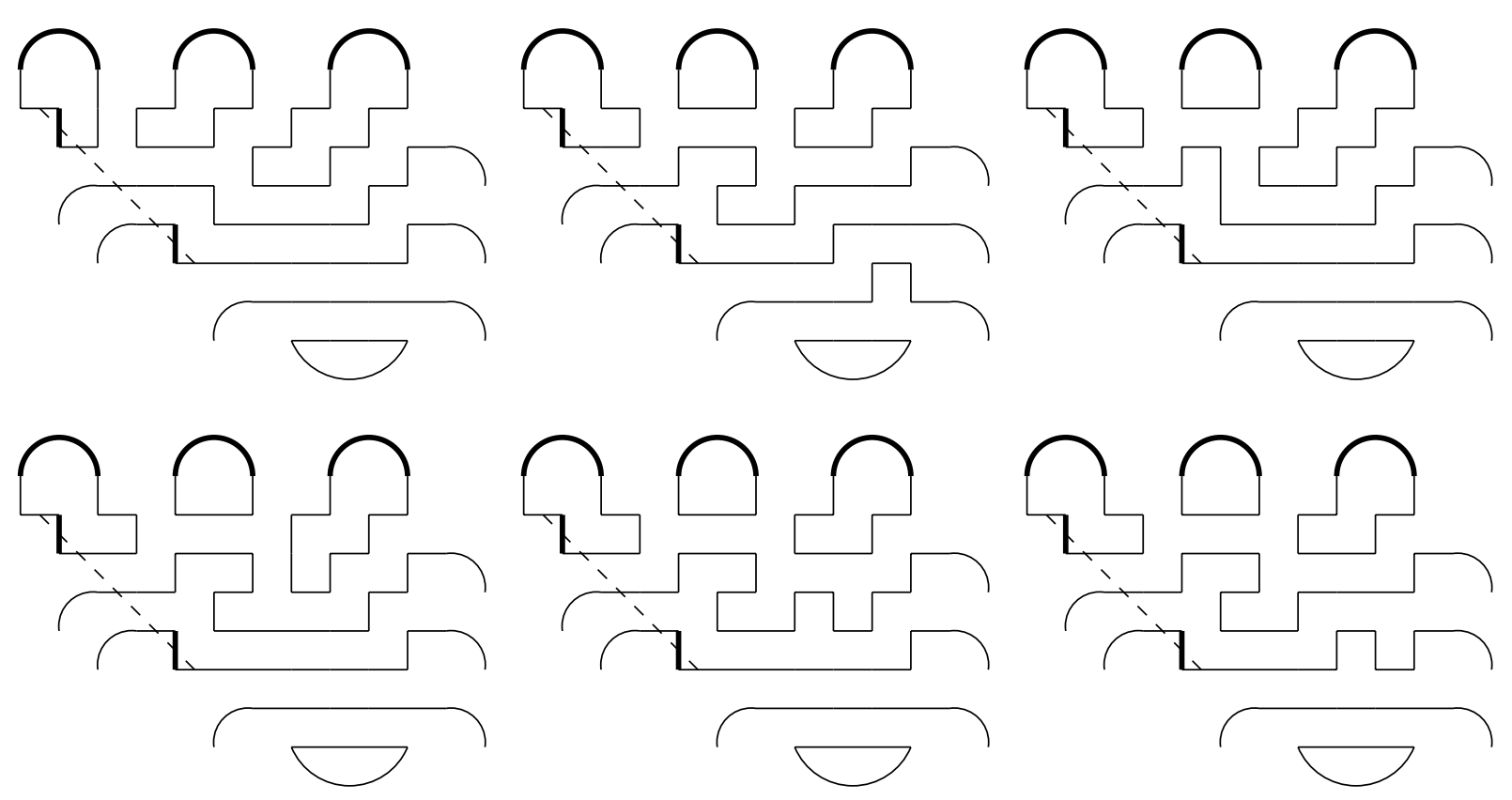

Figure 4.3. The six configurations corresponding to $X=\{1 \leftrightarrow 2,3 \leftrightarrow 4,5 \leftrightarrow$ $6\}$ and $\mathcal{E}=\{1,4\}$

We have $\lambda(X)=(2,1)$ and $\lambda(\mathcal{E})=(1,1)$. In particular, $\lambda(\mathcal{E}) \subseteq \lambda(X)$. The next lemma shows that this is not an accident.

Lemma 4.1. Let $X$ be a non-crossing matching with $d$ arches and let $\mathcal{E}$ be a subset of $\{1,2, \ldots, 2 d-2\}$ consisting of $d-1$ elements.

(1) If $\lambda(\mathcal{E}) \nsubseteq \lambda(X)$, then $a_{X}(\mathcal{E})=0$.

(2) If $\lambda(\mathcal{E})=\lambda(X)$, then $a_{X}(\mathcal{E})=1$.

Proof. This follows from Corollary 7.4.(1) and (3).

Equipped with this lemma, we are now able to prove the first main result of this paper.

Theorem 4.2. Let $X$ be a non-crossing matching with $d$ arches, and let $m \geq 3 d$. Then

$$
A_{X}(m)=S S Y T(\lambda(X), m-2 d+1)+\sum_{\mathcal{E}: \lambda(\mathcal{E}) \varsubsetneqq \lambda(X)} a_{X}(\mathcal{E}) \cdot S S Y T(\lambda(\mathcal{E}), m-2 d+1)
$$

Proof. Let us fix $d-1$ edges from $\left\{e_{1}, e_{2}, \ldots, e_{2 d-2}\right\}$, encoded by the set $\mathcal{E}$. In view of Lemma 4.1, it suffices to show that the number of configurations on the left of the segment $\xi_{1}$ (more precisely, in the region $A K J D E$; see Figure 4.1) which, out of $\left\{e_{1}, e_{2}, \ldots, e_{2 d-2}\right\}$, occupy exactly the edges encoded by $\mathcal{E}$ is equal to $\operatorname{SSYT}(\lambda(\mathcal{E}), m-2 d+1)$. To do so, we proceed in a way similar to the proof of the main results in [2]. That is, we translate the problem of enumerating the latter FPL configurations into a problem of enumerating rhombus tilings.

We say that a vertex is free if it belongs to exactly one fixed edge. We draw a triangle around any free vertex in the region $A K J D E$ in such a way that two free vertices are 


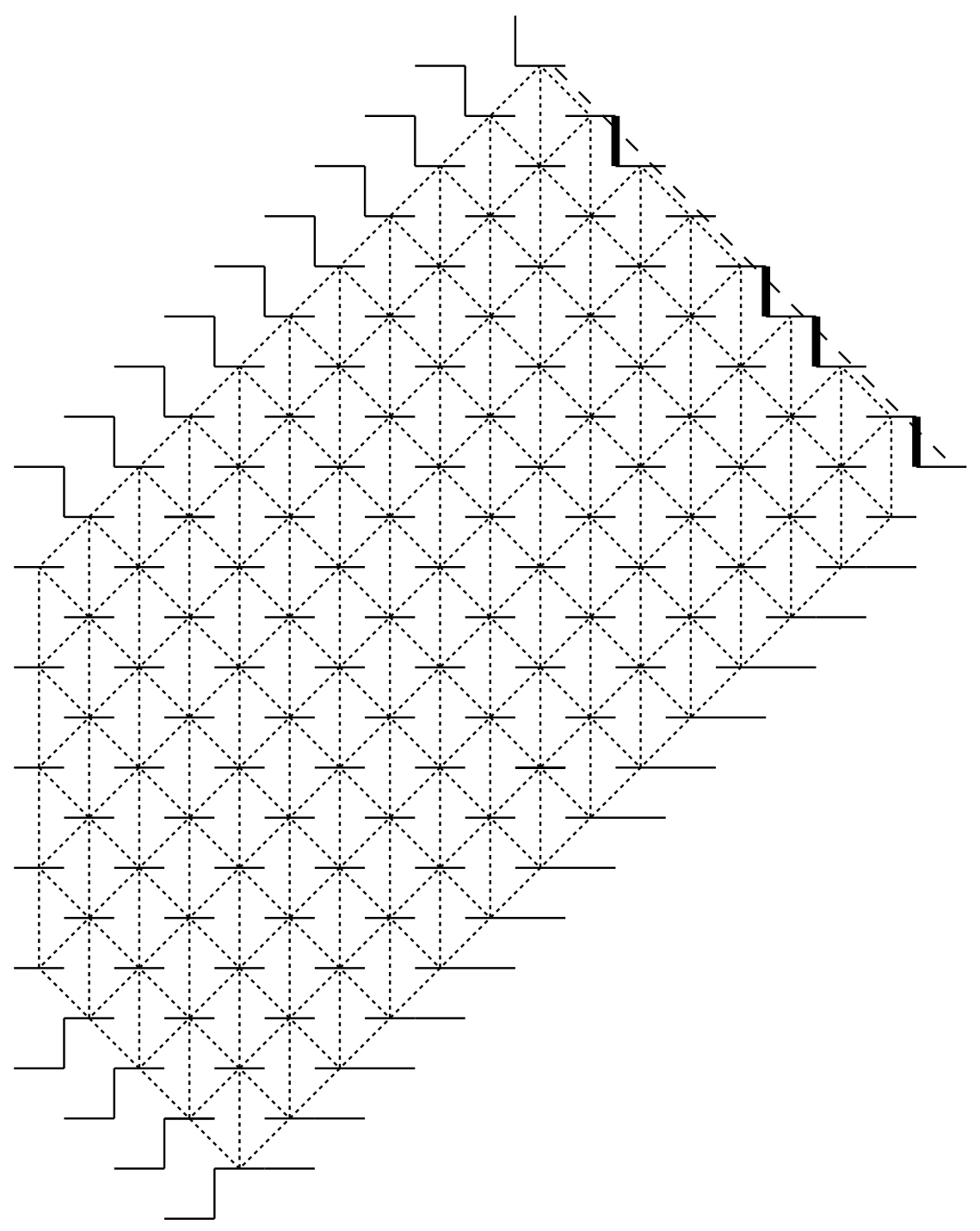

Figure 4.4. Drawing triangles

neighbours if and only if the corresponding triangles share an edge. In the case which is illustrated in Figure 4.1, this leads to the picture in Figure 4.4.

Now we make a deformation of the obtained set of triangles in such a way that all the internal angles become $60^{\circ}$. As a result, we obtain the region $R(\lambda(\mathcal{E}), d-1, m-3 d+2)$ defined in Section 2.5, see Figure 4.5. As in [2], it is not difficult to see that the FPL configurations in the region $A K J D E$ are in bijection with the rhombus tilings of the region $R(\lambda(\mathcal{E}), d-1, m-3 d+2)$. Indeed, to go from a rhombus tiling to the corresponding FPL configuration, for every rhombus in the tiling one connects the free vertices which are in the interior of the two triangles forming the rhombus by an edge. Hence the result follows from Theorem 2.2. 


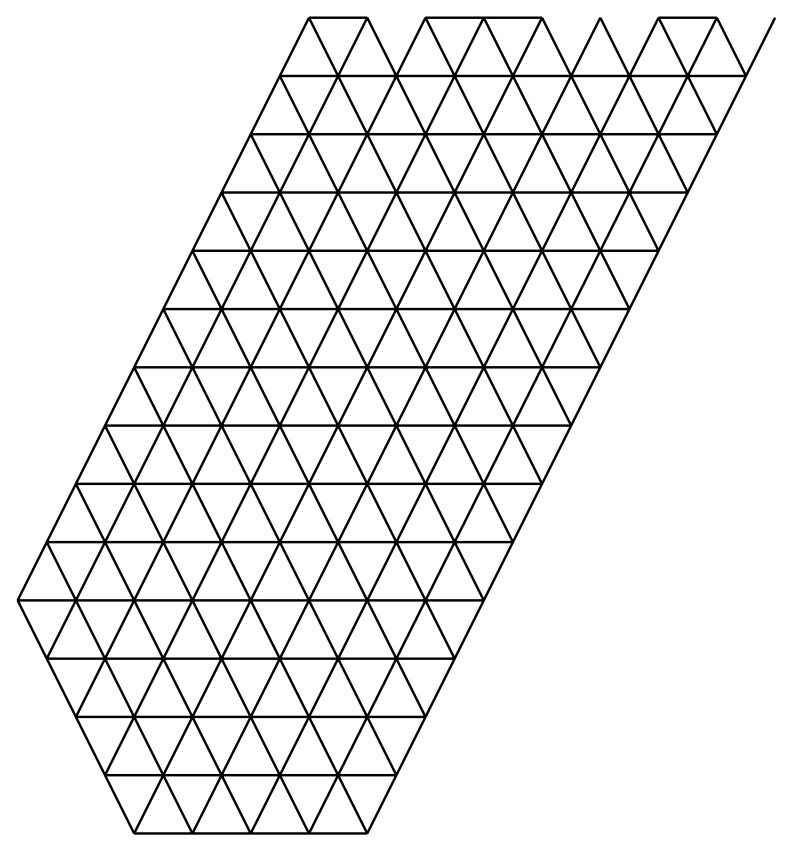

FIGURE 4.5. Getting the regions $R(\mathcal{E}, m+3 d-2)$

Zuber's Conjecture 1.1, in the case that $m \geq 3 d$, is now a simple corollary of the above theorem.

Proof of Conjecture 1.1 for $m \geq 3 d$. The polynomiality in $m$ of $A_{X}(m)$ is obvious from (4.1) and (2.2). The assertion about the integrality of the coefficients of the "numerator" polynomial $P_{X}(m)$ follows from the simple fact that the hook product $\prod_{u \in \lambda} h_{u}$ is a divisor of $|\lambda|$ ! for any partition $\lambda$. Finally, to see that the leading coefficient of $P_{X}(m)$ is $\operatorname{dim}(\lambda(X))$, one first observes that the leading term in (4.1) appears in the term $S S Y T(\lambda(X), m-2 d+1)$. The claim follows now by a combination of $(2.2)$ and $(2.1)$.

\section{Proof of Conjecture 1.1 for Small $m$}

To prove Conjecture 1.1 for $m<3 d$, we choose a different placement of the matching $X \cup m$, namely, we place $X$ around the top-right corner of the square $Q_{n}$. To be precise, we place $X \cup m$ so that the arches corresponding to $X$ occupy the external links labelled $n-2 d+2, n-2 d+4, \ldots, n+2 d$, see Figure 5.1 for an example where $n=28, d=7$, and $m=21$. (There, the positioning of the matching $X$ is indicated by the black hook. The edges in the interior of the square grid should be ignored at this point.) In fact, the figure shows an example where $m \geq 2 d$, and, strange as it may seem, this is what we shall assume in the sequel. Only at the very end, we shall get rid of this assumption.

We now apply again Lemma 3.1 to determine the edges which are occupied by each FPL configuration with associated matching $X \cup m$. As a result, there are fixed edges along zig-zag paths in the upper-left and the lower-right corner of the square grid, while in a pentagonal region located diagonally from lower-left to upper-right every vertex is 


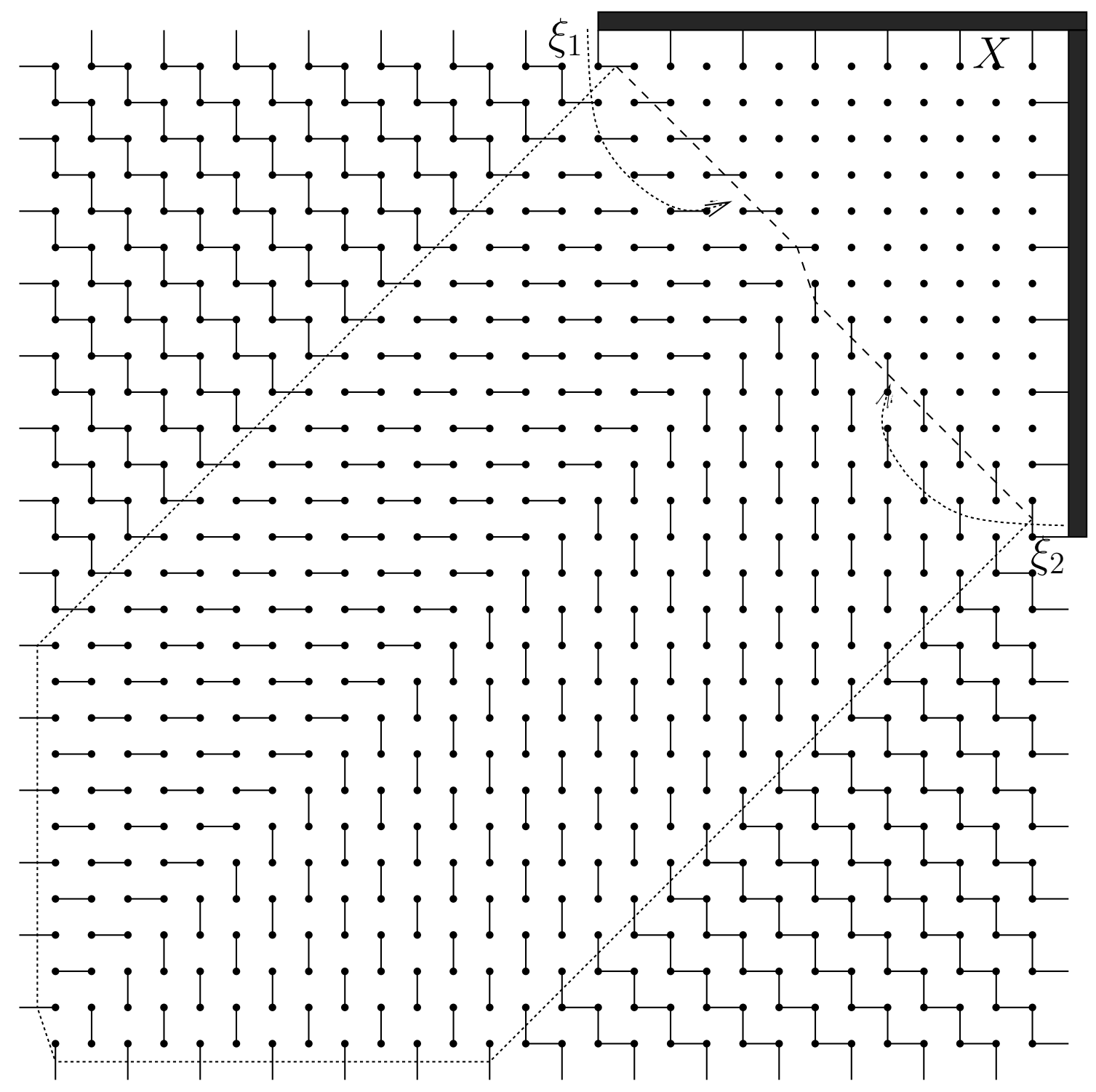

FiguRE 5.1. A different placement of the matching around the grid

on exactly one fixed edge, as indicated in Figure 5.1. (There, the pentagonal region is indicated by the dashed lines.) More precisely, the pentagonal region decomposes into two halves: in the upper-left half every other horizontal edge is taken by any FPL configuration, whereas in the lower-right half it is every other vertical edge which is taken by any FPL configuration.

Now we are argue similarly to the proof of Theorem 4.2. Along the upper-right border, we mark the segments $\xi_{1}$ (the upper-left half; see Figure 5.1) and $\xi_{2}$ (the lower-right half). Let $\left\{e_{1}, e_{2}, \ldots, e_{d-2}\right\}$ be the set of vertical edges which are crossed by $\xi_{1}$, and let $\left\{f_{1}, f_{2}, \ldots, f_{d-1}\right\}$ be the set of vertical edges which are crossed by $\xi_{2}$. In Figure 5.4 these 
edges are marked in bold face. (Compare with Figures 4.1 and 4.4.) Since any loop which enters the triangular region in the upper-right corner of the square grid (that is, the region to the right of the segments $\xi_{1}$ and $\xi_{2}$ ) must necessarily also leave it again, any FPL configuration with associated matching $X \cup m$ occupies a subset of $\left\{e_{1}, e_{2}, \ldots, e_{d-2}\right\}$, encoded by $\mathcal{E}$ as before, and a subset of $\left\{f_{1}, f_{2}, \ldots, f_{d-1}\right\}$, encoded by $\mathcal{F}$, such that $|\mathcal{E}|=|\mathcal{F}|-1$. Once a choice of $\mathcal{E}$ and $\mathcal{F}$ is made, the number of FPL configurations which cover exactly the vertical edges encoded by $\mathcal{E}$ and $\mathcal{F}$ decomposes into the product of the number of possible configurations in the pentagonal region times the number of possible configurations in the triangular region in the upper-right corner of the square grid. Let us denote the former number by $N(\mathcal{E}, \mathcal{F}, m, d)$, and the latter by $c(\mathcal{E}, \mathcal{F})$. Writing, as before, $A_{X}(m)$ for the total number of FPL configurations with associated matching $X \cup m$, we have

$$
A_{X}(m)=\sum_{\mathcal{E}, \mathcal{F}} c(\mathcal{E}, \mathcal{F}) N(\mathcal{E}, \mathcal{F}, m, d)
$$

where the sum is over all possible choices of $\mathcal{E} \subseteq\{1,2, \ldots, d-2\}$ and $\mathcal{F} \subseteq\{1,2, \ldots, d-1\}$ such that $|\mathcal{E}|=|\mathcal{F}|-1$. In the next lemma, we record the properties of the numbers $N(\mathcal{E}, \mathcal{F}, m, d)$ which will allow us to conclude the proof of Conjecture 1.1 for $m<3 d$.

Lemma 5.1. For $m \geq 2 d$, we have

(1) The number $N(\mathcal{E}, \mathcal{F}, m, d)$ is a polynomial in $m$.

(2) As a polynomial in $m, m$ divides $N(\mathcal{E}, \mathcal{F}, m, d)$ for all $\mathcal{E}$ and $\mathcal{F}$, except if $\mathcal{E}=$ $\{1,2, \ldots, d-2\}$ and $\mathcal{F}=\{1,2, \ldots, d-1\}$, in which case $N(\mathcal{E}, \mathcal{F}, m, d)=1$.

Proof. Our aim is to find a determinantal expression for $N(\mathcal{E}, \mathcal{F}, m, d)$. To do so, we proceed as in the proof of Theorem 4.2, that is, we map the possible configurations in the pentagonal region bijectively to rhombus tilings of a certain region in the regular triangular lattice. As in the preceding proof, we draw triangles around free vertices (where "free" has the same meaning as in that proof) in such a way that two free vertices are neighbours if and only if the corresponding triangles share an edge. The region in the regular triangular lattice which we obtain for the pentagonal region of Figure 5.1 is shown in Figure 5.2. It is a hexagon with bottom side of length $d-1$, lower-left side of length $d-1$, upper-left side of length $m-d+2$, top side of length $d-2$, upper-right side of length $d$, and lower-right side of length $m-d+1$. However, depending on the choice of $\mathcal{E}$ and $\mathcal{F}$, along the top side and along the upper-right side there are some notches (that is, triangles of unit side length which are missing, as was the case for the region $R(\mathcal{E}, m-3 d+1)$ obtained in the proof of Theorem 4.2; compare with Figure 4.5). In Figure 5.2, the possible places for notches are labelled $\left\{e_{1}, e_{2}, \ldots, e_{d-2}\right\}$, respectively $\left\{f_{1}, f_{2}, \ldots, f_{d-1}\right\}$. The place labelled $f_{d}$ cannot be the place of a notch, and the number of notches out of $\left\{f_{1}, f_{2}, \ldots, f_{d-1}\right\}$ must be exactly by 1 larger than the number of notches out of $\left\{e_{1}, e_{2}, \ldots, e_{d-2}\right\}$. An example of a choice of notches for $d=5, m=7, \mathcal{E}=\{2\}$ and $\mathcal{F}=\{1,4\}$ (filled by a rhombus tiling of the resulting region) is shown on the left in Figure 5.3.

Thus, the number $N(\mathcal{E}, \mathcal{F}, m, d)$ is equal to the number of rhombus tilings of this hexagonal region with notches. To get a formula for the number of rhombus tilings, we 


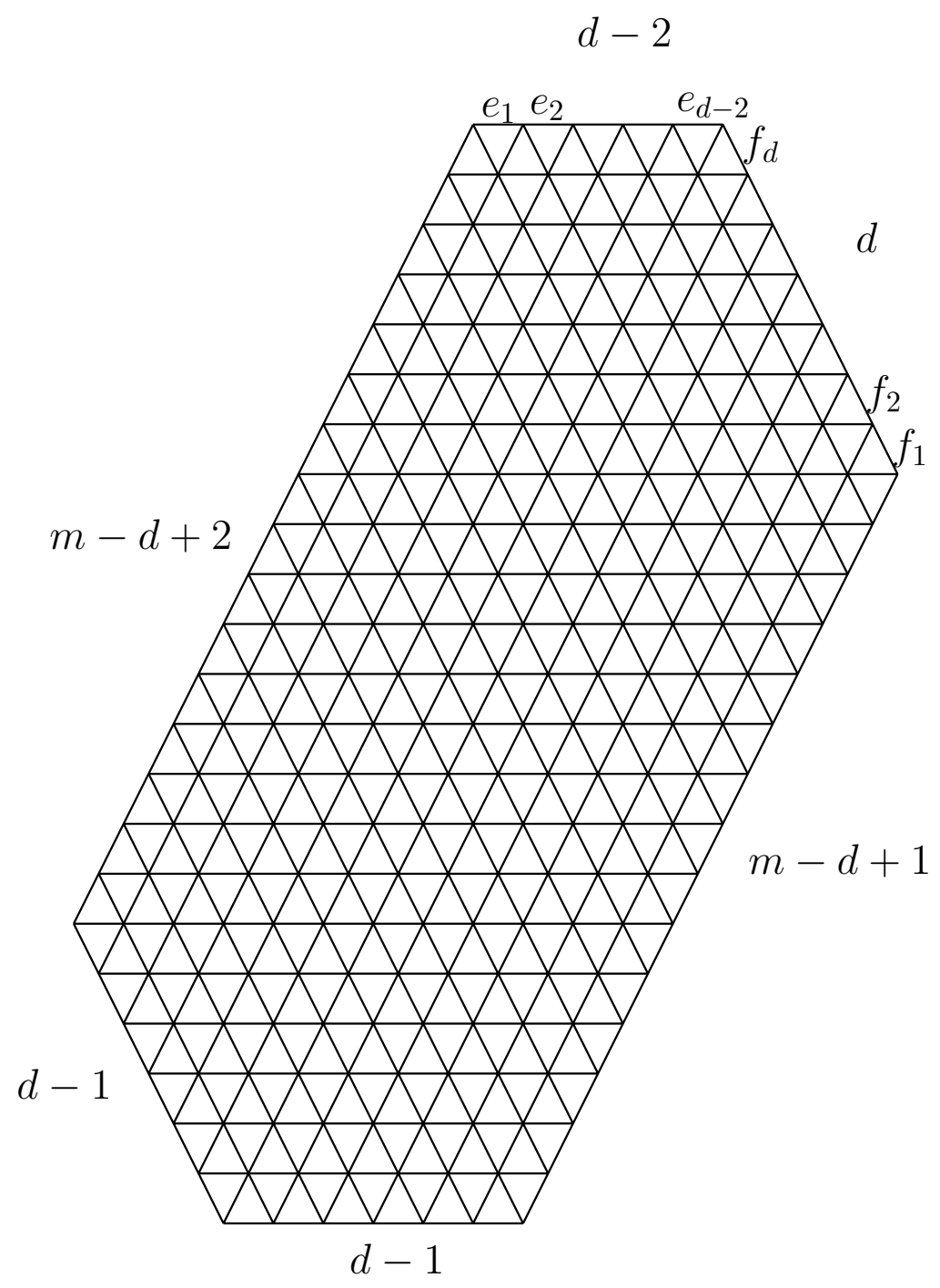

FiguRE 5.2. The region to be tiled

apply the standard bijection between rhombus tilings and families of non-intersecting lattice paths (see, e.g., $[3,4]$ ). The bijection is obtained as follows. One places vertices in each of the mid-points of edges along the bottom-left side of the region, and as well in each mid-point along the downward edges which form part of a notch on the top of the region, and along the downward edges along the top-right side of the region. (In Figure 5.3 these midpoints are marked in boldface.) The vertices of the lower-left edges are subsequently connected to the vertices on top and top-right by paths, by connecting the mid-points of opposite downward edges in each rhombus of the tiling, see again Figure 5.3. Clearly, by construction, the paths are non-intersecting. Subsequently, the paths are slightly rotated, and deformed so that they become rectangular paths. Thus, one obtains families of paths with starting points $A_{i}=(-i, i), i=1,2, \ldots, d-1$, and with end points a 
subset of cardinality $d-1$ from the points $\left\{E_{1}, E_{2}, \ldots, E_{d-2}\right\} \cup\left\{F_{1}, F_{2}, \ldots, F_{d}\right\}$, where $E_{i}=(-d+i, m+1), i=1, \ldots, d-2$, and $F_{j}=(d-j-1, m-d+j+1), j=1,2, \ldots, d$. The point $F_{d}$ must be among the end points. The family of paths which results from our example rhombus tiling is shown on the right of Figure 5.3.
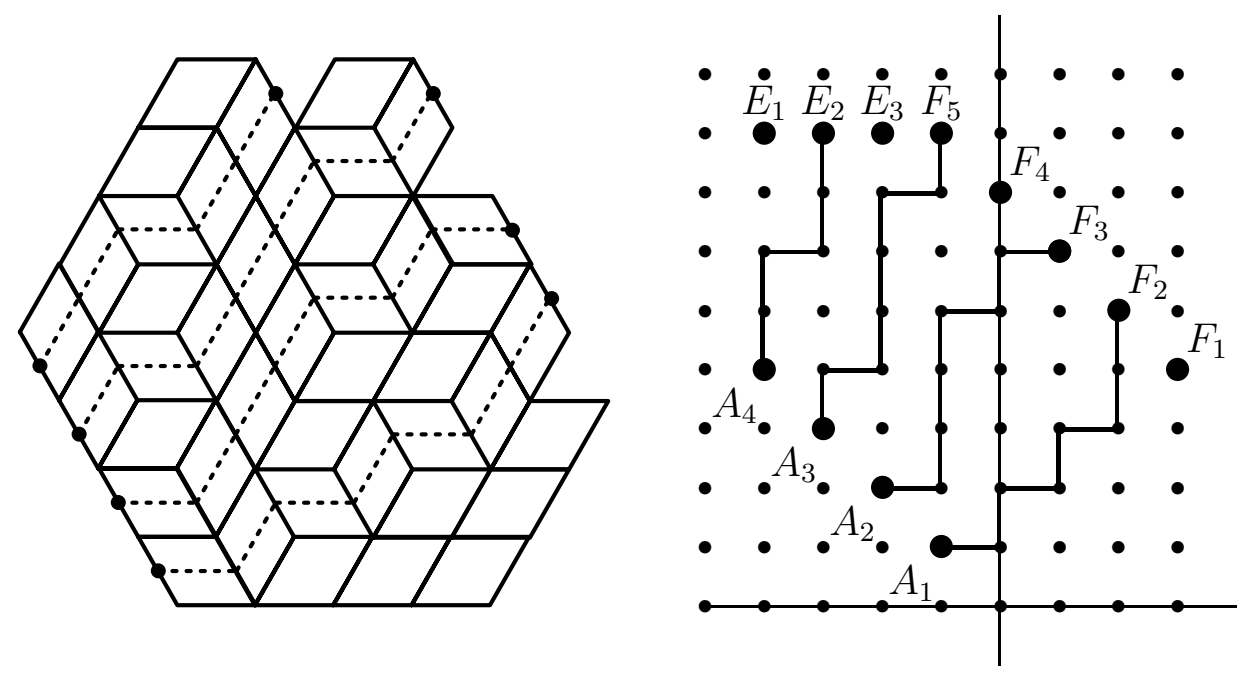

FIGURE 5.3. A rhombus tiling of the region with notches, and its corresponding family of non-intersecting lattice paths

Now we can apply Lemma 2.3 to obtain a determinant for the number of rhombus tilings. The number of paths from a starting point $A_{i}$ to an end point, which forms an entry in the determinant in $(2.3)$, is equal to $\left(\begin{array}{c}m+1+j-d \\ j+i-d\end{array}\right)$ if the end point is $E_{j}$, and it is $\left(\begin{array}{c}m \\ d-j+i-1\end{array}\right)$ if the end point is $F_{j}$. In both cases, this is a polynomial in $m$, and thus claim (1) follows.

To prove claim (2), we observe that $m$ divides the second of the above binomial coefficients, $\left(\begin{array}{c}m \\ d-j+i-1\end{array}\right)$, as long as $d-j+i-1>0$. However, this will be always the case, except if $i=1$ and $j=d$. In particular, if we choose an $F_{j}$ with $j<d$ as an end point, all the entries in the column corresponding to $F_{j}$ in the determinant (2.3) will be divisible by $m$, and, hence, the determinant itself. Thus, the only case where the determinant is not divisible by $m$ is the one where the set of end points is $\left\{E_{1}, E_{2}, \ldots, E_{d-2}, F_{d}\right\}$. This corresponds to the choice $\mathcal{E}=\{1,2, \ldots, d-2\}$ and $\mathcal{F}=\{1,2, \ldots, d-1\}$. In addition, in that case the determinant in (2.3) is equal to 1 because it is the determinant of a triangular matrix with 1s on the antidiagonal. This completes the proof of the lemma.

We are now in the position to complete the proof of Conjecture 1.1 for $m<3 d$. We wish to prove that the polynomial which results from the right-hand side of (5.1) by substituting the determinantal formula for $N(\mathcal{E}, \mathcal{F}, m, d)$ obtained in the preceding proof of Lemma 5.1 gives the number of FPL configurations under consideration for all $m \geq 0$. As we remarked earlier, the arguments so far show only that this is indeed the case for $m \geq 2 d$. 


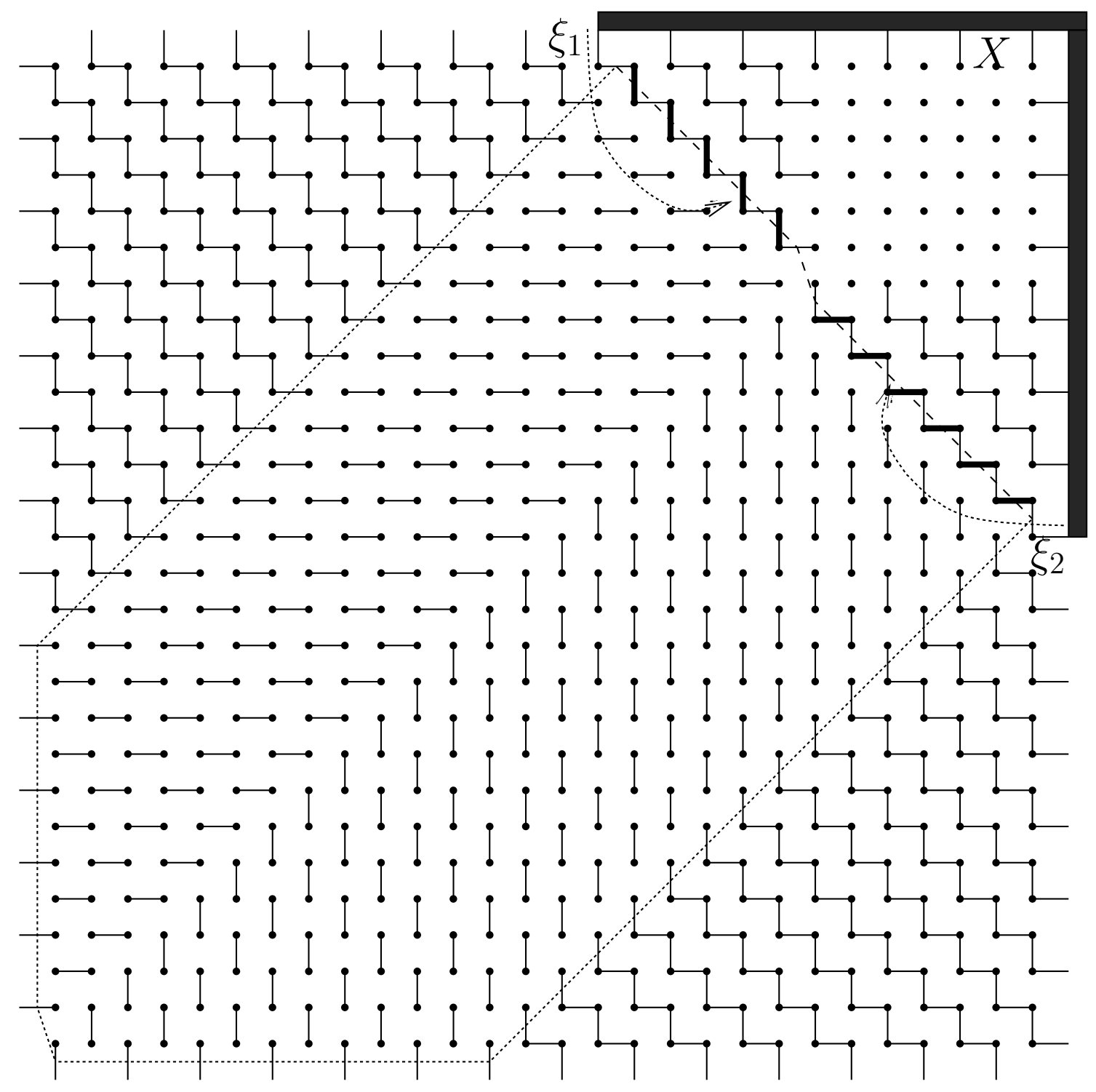

Figure 5.4. Fixed edges for $\mathcal{E}=\{1, \ldots, d-2\}$ and $\mathcal{F}=\{1, \ldots, d-1\}$

We verify next that this is also the case for $m=0$. If we put $m=0$ in the right-hand side of (5.1) (with the afore-mentioned substitution for $N(\mathcal{E}, \mathcal{F}, m, d)$ ), then, by Lemma 5.1, the only term which survives is the one for $\mathcal{E}=\{1,2, \ldots, d-2\}$ and $\mathcal{F}=\{1,2, \ldots, d-1\}$. In addition, in that case we have $N(\mathcal{E}, \mathcal{F}, m, d)=1$. Thus, if $m=0$, the expression in (5.1) is equal to $c(\mathcal{E}, \mathcal{F})$, the number of possible configurations in the triangular region in the upper-right corner of the square grid. Figure 5.4 shows what happens inside this triangular region for this choice of $\mathcal{E}$ and $\mathcal{F}$ : from the external links occupied by the matching $X$ there propagate zig-zag lines of fixed edges into the interior, so that only a square region with side length $d-1$ remains undetermined. Thus, the number of possible 
FPL configurations inside this triangular region is indeed equal to the number of FPL configurations with associated matching $X$ (that is, FPL configurations on the square $Q_{d}$ with associated matching $X$ ), which is exactly what we wanted to prove.

While it seems that we have still a large gap (namely the values of $m$ between 1 and $2 d-1)$ to overcome, the assertion now follows: let $H_{X}(m)$ denote the polynomial on the right-hand side of (5.1). By the above arguments we know that, for an arbitrary nonnegative integer $m$, the number $A_{X}(m)=A_{X \cup m}(0)$ of FPL configurations with associated matching $X \cup m$ is equal to $H_{X \cup m}(0)$. Furthermore, for any non-negative integer $s$ we have $A_{X \cup m}(s)=A_{X}(m+s)$. By the preceding arguments, if $s$ is sufficiently large, we have also $A_{X \cup m}(s)=H_{X \cup m}(s)$ and $A_{X}(m+s)=H_{X}(m+s)$. However, it also follows from the preceding arguments that $H_{X \cup m}(s)$ and $H_{X}(m+s)$ are polynomials in $s$. Since they agree for an infinite number of values of $s$, they must be identical. In particular, $A_{X \cup m}(0)=H_{X \cup m}(0)=H_{X}(m)$. Thus, the number $A_{X \cup m}(0)$ of FPL configurations with associated matching $X \cup m$ is indeed given by the same polynomial for any $m$. It must necessarily be equal to the polynomial found in Section 4 (see the last paragraph of that section). Conjecture 1.1 is now completely proved.

\section{Proof of Conjecture 1.2 For $m$ LARge Enough}

In this section we show how the ideas developed in the proof of Theorem 4.2 can be extended to prove Conjecture 1.2 for large enough $m$.

Let $X$ and $Y$ be two non-crossing matchings with $d$ and $e$ arches, respectively. Without loss of generality, we may suppose that $d \geq e$. We choose to place the matching $X \cup m \cup Y$ in Conjecture 1.2 in such a way that, again, the set of arches of $X$ appear on the very right of the upper side of the grid $Q_{n}=Q_{d+e+m}$, see Figure 6.1 for a schematic picture, that is, we place these arches on the external links labelled $n-4 d+2, n-4 d+4, \ldots, n-2, n$. In order to guarantee that $X$ has place along the upper side of the square grid, we must assume that $m \geq 3 d-e$.

Next we determine the set of fixed edges using Lemma 3.1. For convenience (the reader should consult Figure 6.2 while reading the following definitions; it contains an example with $n=15, d=3, e=2$ and $m=10$ ), we let $A, B, C, F, G, D, E$ (in this order!) be the border vertices of the external links labelled $n-4 d+3, n-1, n+2 d+2 e-2$, $-n-2 d-2 e+3,-n-2 d+2 e-1,-n+2 d-2 e+2,-n+4 d-2$, respectively, we let $K$ be the point in the interior of $Q_{n}$ which makes $A B K$ into a rectangular isosceles triangle, with the right angle at $K$, we let $M$ be the analogous point which makes $F G M$ into a rectangular isosceles triangle, with the right angle at $M$, we let $J$ be the intersection point of the line connecting $F$ and $M$ and the line connecting $B$ and $K$, and we let $L$ be the intersection point of the line connecting $G$ and $M$ and the line connecting $A$ and $K$. We state the result of the application of Lemma 3.1 to the current case in form of the following lemma.

Lemma 6.1. The region of fixed edges of the FPL configurations corresponding to the matching $X \cup m \cup Y$ contains all the edges indicated in Figure 6.2, that is:

(1) all the horizontal edges in the rectangular region JKLM, 


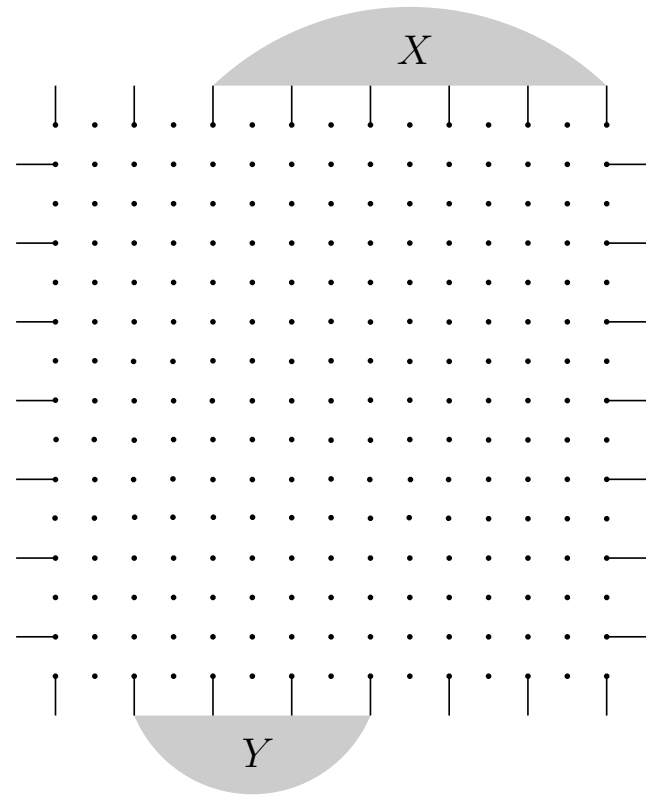

FiguRE 6.1. Placing the matching around the grid

(2) every other horizontal edge in the L-shaped region AKJMGDE as indicated in the figure,

(3) every other horizontal edge in the region BCFMLK as indicated in the figure,

(4) the zig-zag lines in the corner regions above the line EA, respectively below the lines $D G$ and $F C$, as indicated in the figure.

Let $\xi_{1}$ be the segment which connects the point which is half a unit to the left of $A$ and the point which is half a unit to the left of $K$, and let $\xi_{2}$ be the segment which connects the point which is half a unit to the right of $K$ with the point which is half a unit to the right of $B$, see Figure 6.2. Similarly, let $\eta_{1}$ be the segment which connects the point which is half a unit to the left of $G$ and the point which is half a unit to the left of $M$, and let $\eta_{2}$ be the segment which connects the point which is half a unit to the right of $M$ with the point which is half a unit to the right of $F$, see again Figure 6.2. There are $2 d-2$ vertical edges which cross $\xi_{1}$, and the same is true for $\xi_{2}$, and there are $2 e-2$ vertical edges which cross $\eta_{1}$, and the same is true for $\eta_{2}$.

The next lemma says that, also in this more general situation, it is exactly one half of the vertical edges which cross $\xi_{1}, \xi_{2}, \eta_{1}$, and $\eta_{2}$, respectively, which are taken by any FPL configuration with associated matching $X \cup m \cup Y$.

Lemma 6.2. Any FPL configuration with associated matching $X \cup m \cup Y$, occupies exactly $d-1$ vertical edges crossing the segments $\xi_{1}$, the same being true for $\xi_{2}$, and it occupies exactly $e-1$ vertical edges crossing the segments $\eta_{1}$, the same being true for $\eta_{2}$.

Proof. This follows from Corollary 7.4.(2). 


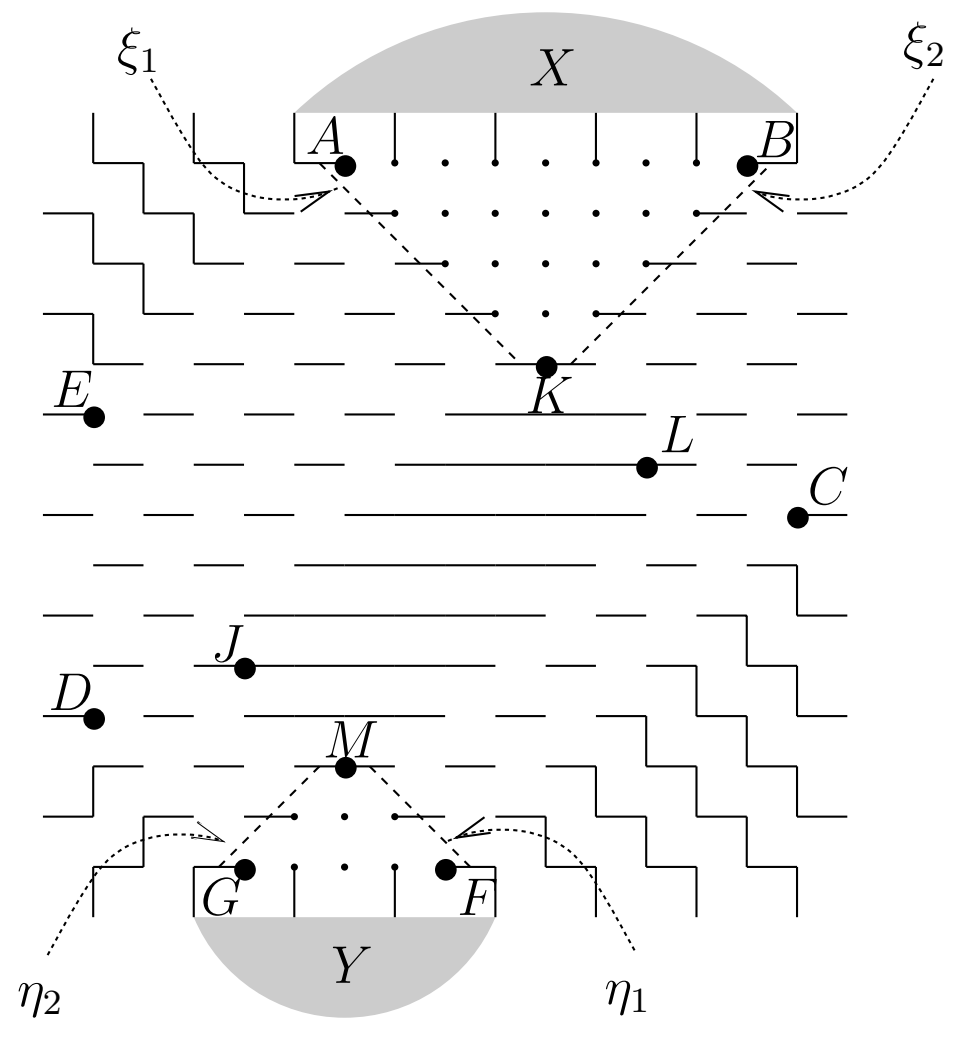

Figure 6.2. The fixed edges

Hence, any such choice of edges as described in Lemma 6.2 can be encoded, as before, by four sets $\mathcal{E}_{1}, \mathcal{E}_{2}, \mathcal{F}_{1}, \mathcal{F}_{2}$, the sets $\mathcal{E}_{1}$ and $\mathcal{E}_{2}$ being subsets of $\{1,2, \ldots, 2 d-2\}$, and the sets $\mathcal{F}_{1}$ and $\mathcal{F}_{2}$ being subsets of $\{1,2, \ldots, 2 e-2\}$. We make the convention that $\mathcal{E}_{1}$ encodes the set of vertical edges crossing the segment $\xi_{1}, \mathcal{E}_{2}$ encodes the set of vertical edges crossing the segment $\xi_{2}, \mathcal{F}_{1}$ the set of edges crossing the segment $\eta_{1}$ and finally $\mathcal{F}_{2}$ the set of edges crossing the segment $\eta_{2}$. We denote by $a_{X}\left(\mathcal{E}_{1}, \mathcal{E}_{2}\right)$ the number of FPL configurations in the triangular region $A B K$, which "obey" the matching $X$, which, out of the edges crossing the segment $\xi_{1}$, occupy exactly those determined by $\mathcal{E}_{1}$, and which, out of the edges crossing the segment $\xi_{2}$, occupy exactly those determined by $\mathcal{E}_{2}$. Note that, by Corollary 7.4.(1), $a_{X}\left(\mathcal{E}_{1}, \mathcal{E}_{2}\right)$ can only be non-zero if $\lambda\left(\mathcal{E}_{1}\right) \subseteq \lambda(X)$ and $\lambda\left(\mathcal{E}_{2}\right) \subseteq \lambda(X)^{\prime}$, where $\lambda(X)^{\prime}$ denotes the Ferrers diagram conjugate to $\lambda(X)$. For the latter assertion, we use the following trivial observation: Let $X$ be a non-crossing matching with $d$ arches, and let $X^{r}$ be the "reversed" matching, that is, whenever $i$ is matched with $j$ in $X, 2 d-i+1$ is matched with $2 d-j+1$ in $X^{r}$. Then, under the correspondence between matchings and Ferrers diagrams described in Section 2.4, we have $\lambda\left(X^{r}\right)=\lambda(X)^{\prime}$.

In order to proceed, we need to introduce a region in the triangular lattice which is parametrized by two Ferrers diagram and four non-negative integers. Let $h, k, d, e$ be non-negative integers, and let $\lambda \subseteq\left(d^{d}\right)$ and $\mu \subseteq\left(e^{e}\right)$ be two Ferrers diagrams. Then we 


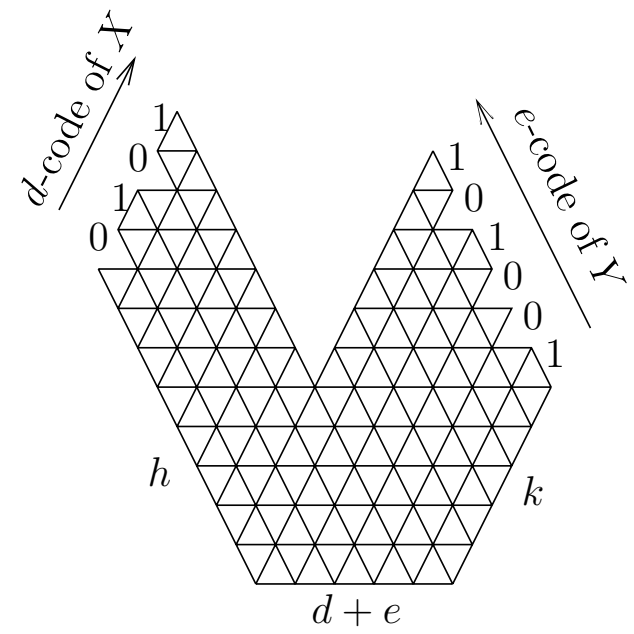

Figure 6.3. The region $R(X, Y, d, e, h, k)$

define $R(X, Y, d, e, h, k)$ to be the $\mathrm{V}$-shaped region (the reader should consult Figure 6.3 while reading the following description; the figure illustrates $R(X, Y, d, e, h, k)$ for $d=2$, $e=3, h=8, k=5, \lambda=(1,0)$, and $\mu=(2,1,1))$ with base side of length $d+e$, left side $h$, followed by a side in direction north-east of length $2 d$ with notches which will be explained in just a moment, a V-shaped "valley," a side in direction south-east of length $2 e$ with notches which will be explained in just a moment, and finally a right side of length $k$. To determine the notches along the side in direction north-east, we read the $d$-code of $\lambda$ and while, at the same time, moving along the side from south-west to north-east, we put a notch whenever we read a 0 , and we leave a horizontal piece whenever we read a 1 . The notches along the side in direction south-east are determined in a similar way from the $e$-code of $\mu$, however, while reading the $e$-code, we move along the side from south-east to north-west. Let $r(X, Y, d, e, h, k)$ be the number of rhombus tilings of $R(X, Y, d, e, h, k)$.

With the above notation, we have the following expansion for the number $A_{X, Y}(m)$ of FPL configurations with associated matching $X \cup m \cup Y$.

Theorem 6.3. Let $X$ and $Y$ be two non-crossing matchings with $d$ and $e$ arches, respectively. Then, for $m \geq 3 d-e$,

$$
\begin{aligned}
A_{X, Y}(m)= & \sum_{\mathcal{E}_{1}, \mathcal{E}_{2}, \mathcal{F}_{1}, \mathcal{F}_{2}} a_{X}\left(\mathcal{E}_{1}, \mathcal{E}_{2}\right) a_{Y}\left(\mathcal{F}_{1}, \mathcal{F}_{2}\right) \\
& \cdot r\left(\lambda\left(\mathcal{E}_{1}\right), \lambda\left(\mathcal{F}_{2}\right), d-1, e-1, m-3 d+e+2,2 d-2 e+1\right) \\
& \cdot r\left(\lambda\left(\mathcal{F}_{1}\right), \lambda\left(\mathcal{E}_{2}\right), e-1, d-1, m-d-e+2,1\right),
\end{aligned}
$$

where the sum is taken over all subsets $\mathcal{E}_{1}, \mathcal{E}_{2}$ of $\{1,2, \ldots, 2 d-2\}$ and all subsets $\mathcal{F}_{1}, \mathcal{F}_{2}$ of $\{1,2, \ldots, 2 e-2\}$ such that $\lambda\left(\mathcal{E}_{1}\right) \subseteq \lambda(X), \lambda\left(\mathcal{E}_{2}\right) \subseteq \lambda(X)^{\prime}, \lambda\left(\mathcal{F}_{1}\right) \subseteq \lambda(Y)$, and $\lambda\left(\mathcal{F}_{2}\right) \subseteq$ $\lambda(Y)^{\prime}$. 
Proof. Let us fix $d-1$ edges which cross $\xi_{1}, d-1$ edges which cross $\xi_{2}, e-1$ edges which cross $\eta_{1}, e-1$ edges which cross $\eta_{2}$, encoded by the sets $\mathcal{E}_{1}, \mathcal{E}_{2} \subseteq\{1,2, \ldots, 2 d-2\}$ of cardinality $d-1$ and by the sets $\mathcal{F}_{1}, \mathcal{F}_{2} \subseteq\{1,2, \ldots, 2 e-2\}$ of cardinality $e-1$, respectively. Clearly, the number of FPL configurations with associated matching $X \cup m \cup Y$ which occupy exactly these vertical edges crossing $\xi_{1}, \xi_{2}, \eta_{1}$, and $\eta_{2}$ decomposes into a product: it equals the number of configurations in the region $A B K$, given by $a_{X}\left(\mathcal{E}_{1}, \mathcal{E}_{2}\right)$, times the number of configurations in the region $F G M$, given by $a_{Y}\left(\mathcal{F}_{1}, \mathcal{F}_{2}\right)$, times the number of configurations in the region $A K J M G D E$, times the number of configurations in the region $F M L K B C$.

In order to compute the number of configurations in the latter two regions, as in the proof of Theorem 4.2, we translate again the problem of enumerating FPL configurations into a problem of enumerating rhombus tilings. If we do this for the region $A K J M G D E$, then, as a result, we have to count all the rhombus tilings of the region $R\left(\lambda\left(\mathcal{E}_{1}\right), \lambda\left(\mathcal{F}_{2}\right), d-\right.$ $1, e-1, m-3 d+e+2,2 d-2 e+1)$, while for the region $F M L K B C$ we arrive at the problem of counting all the rhombus tilings of the region $R\left(\lambda\left(\mathcal{F}_{1}\right), \lambda\left(\mathcal{E}_{2}\right), e-1, d-1, m-d-e+2,1\right)$. This finishes the proof of the theorem.

Our aim is to show that the quantities $r($.$) appearing in (6.1) are polynomials in m$, and that the "dominating" term in the sum in (6.1) comes from choosing $\mathcal{E}_{1}, \mathcal{E}_{2}, \mathcal{F}_{1}, \mathcal{F}_{2}$, such that $\lambda\left(\mathcal{E}_{1}\right)=\lambda(X), \lambda\left(\mathcal{F}_{1}\right)=\lambda(Y)$, and $\lambda\left(\mathcal{E}_{2}\right)=\lambda\left(\mathcal{F}_{2}\right)=\emptyset$. In order to do so, we need several preparatory lemmas.

Lemma 6.4. Let $\lambda$ be a partition contained in $\left(d^{d}\right)$, and let $\mu$ be a partition contained in $\left(e^{e}\right)$. Then the number $r(\lambda, \mu, d, e, h, k)$ of rhombus tilings of the region $R(\lambda, \mu, d, e, h, k)$ is equal to

$$
r(\lambda, \mu, d, e, h, k)=\frac{1}{|\lambda| !} p_{\lambda, \mu, d, e, k}(h),
$$

where $p_{\lambda, \mu, d, e, k}(h)$ is a polynomial of degree $|\lambda|$ with integer coefficients.

Proof. Again, we use the standard bijection between rhombus tilings and non-intersecting lattice paths (cf. the proof of Lemma 5.1). This time, we introduce vertices along the bottom side of the region $R(\lambda, \mu, d, e, h, k)$, and along the horizontal edges which are part of the notches on top-left and top-right of the region (see Figure 6.4, which shows the region $R((1,0),(2,1,1), 2,3,8,5)$ of Figure 6.3$)$. These vertices are then connected by paths, as before. If, finally, the paths are again rotated and slightly deformed so that they become rectangular paths, we obtain families $\left(P_{1}, P_{2}, \ldots, P_{d+e}\right)$ of non-intersecting lattice paths, where the starting points of the paths $P_{i}$ are $A_{i}=(-i, i), i=1,2, \ldots, d+e$, and where their end points are the points $E_{j}=\left(\lambda_{j}-j-e, h+d+e\right), j=1,2, \ldots, d$, and $F_{j}=\left(k-1, \mu_{j}+e-j+1\right), j=1,2, \ldots, e$.

We may now apply Lemma 2.3 to obtain a determinant for the number these families of non-intersecting lattice paths. The number of paths from a starting point $A_{i}$ to an end point, which forms an entry in the determinant in $(2.3)$, is equal to $\left(\begin{array}{c}\lambda_{j}-j+h+d \\ \lambda_{j}-j-e+i\end{array}\right)$ if the end point is $E_{j}$, and it is $\left(\begin{array}{c}k+\mu_{j}+e-j \\ k-1+i\end{array}\right)$ if the end point is $F_{j}$. 

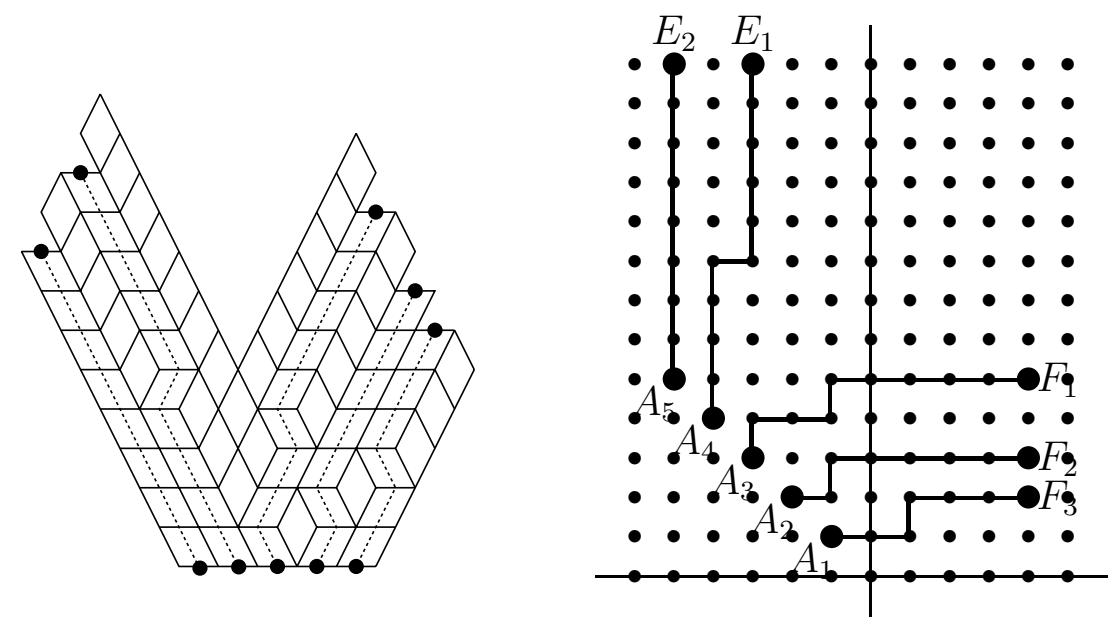

FiguRE 6.4. Non-intersecting lattice paths for a rhombus tiling of $R(\lambda, \mu, d, e, h, k)$

If we expand the determinant (2.3), with these specializations, according to its definition, then we obtain, up to an overall sign,

$$
\sum_{\sigma \in S_{d+e}} \operatorname{sgn} \sigma\left(\prod_{j=1}^{d}\left(\begin{array}{c}
\lambda_{j}-j+h+d \\
\lambda_{j}-j-e+\sigma(j)
\end{array}\right)\right)\left(\prod_{j=d+1}^{d+e}\left(\begin{array}{c}
k+\mu_{j}+e-j \\
k-1+\sigma(j)
\end{array}\right)\right),
$$

where $S_{N}$ denotes the group of permutations of $\{1,2, \ldots, N\}$.

To determine the degree of (6.2) as a polynomial in $h$, we observe that the second product does not contribute, whereas the degree in $h$ of the first product is $\sum_{j=1}^{d}\left(\lambda_{j}-j-\right.$ $e+\sigma(j))=|\lambda|+\sum_{j=1}^{d}(\sigma(j)-j-e)$. The latter sum is maximal exactly if $\sigma(j)=j+e$, $j=1,2, \ldots, d$, in which case it equal to 0 . Thus, the degree of (6.2) as a polynomial in $h$ is indeed $|\lambda|$.

Finally, to show that, if we put (6.2) on the common denominator $|\lambda|$ !, the numerator polynomial has integer coefficients, we observe that the second product in (6.2) is an integer. Thus, it suffices to multiply the first product by $|\lambda|$ !, and show that the result is a polynomial in $h$ with integer coefficients. Indeed, this is

$$
\begin{aligned}
& \text { (6.3) }|\lambda| !\left(\prod_{j=1}^{d}\left(\begin{array}{c}
\lambda_{j}-j+h+d \\
\lambda_{j}-j-e+\sigma(j)
\end{array}\right)\right) \\
& =L !\left(\begin{array}{c}
|\lambda| \\
\lambda_{1}-1-e+\sigma(1), \ldots, \lambda_{d}-d-e+\sigma(d), L
\end{array}\right) \prod_{j=1}^{d}(h+d+e-\sigma(j)+1)_{\lambda_{j}-j-e+\sigma(j)},
\end{aligned}
$$

where $L=\sum_{j=1}^{d}(j+e-\sigma(j))$ (which is non-negative, as we have just shown!), where the Pochhammer symbol $(\alpha)_{s}$ is defined by $(\alpha)_{s}:=\alpha(\alpha+1) \cdots(\alpha+s-1)$, and where for 
$N=N_{1}+N_{2}+\cdots+N_{s}$ the multinomial coefficient is defined by

$$
\left(\begin{array}{c}
N \\
N_{1}, N_{2} \ldots, N_{s}
\end{array}\right)=\frac{N !}{N_{1} ! N_{2} ! \cdots N_{s} !} .
$$

Evidently, the right-hand side in (6.3) is a polynomial in $h$ with integer coefficients.

Lemma 6.5. Let $\lambda$ be a partition contained in $\left(d^{d}\right)$. The number of rhombus tilings of $R(\lambda, \emptyset, d, e, h, k)$ is equal to the number of rhombus tilings of the region $R(\lambda, d, h)$ defined in Section 2.5.

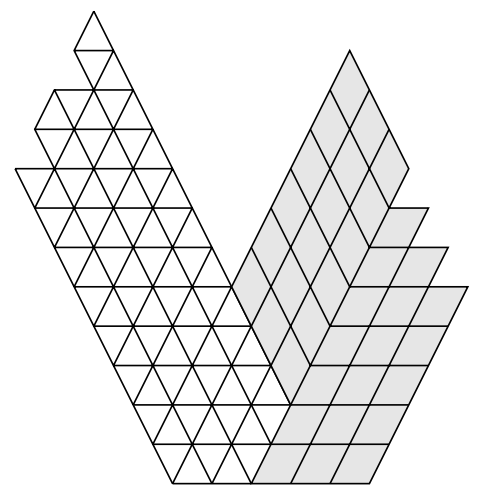

Figure 6.5. The forced rhombi in $R(\lambda, \emptyset, d, e, h, k)$

Proof. The reader should consult Figure 6.5 for an example of a region $R(\lambda, \mu, d, e, h, k)$ where $\mu$ is the empty partition. In that case, there are many "forced" rhombi in the right half of the V-shaped region, that is, rhombi which are common to every rhombus tiling of the region. In the example in Figure 6.5, these rhombi are the ones in the shaded region. Once we remove the forced rhombi, we are left with (a reflected version of) the region $R(\lambda, d, h)$.

Lemma 6.6. Let $X$ be a non-crossing matching with $d$ arches, and let $\mathcal{E}_{1}$ be the subset of $\{1,2, \ldots, 2 d-2\}$ for which $\lambda(X)=\lambda\left(\mathcal{E}_{1}\right)$. Then

$$
a_{X}\left(\mathcal{E}_{1}, \mathcal{E}_{2}\right)= \begin{cases}0, & \text { if } \mathcal{E}_{2} \neq\{1,2, \ldots, d-1\}, \\ 1, & \text { if } \mathcal{E}_{2}=\{1,2, \ldots, d-1\} .\end{cases}
$$

Proof. This follows from the proof of Corollary 7.4.(3).

Remark. The reader should note that the choice of $\mathcal{E}_{2}=\{1,2, \ldots, d-1\}$ means that $\lambda\left(\mathcal{E}_{2}\right)=\emptyset$.

If we use Lemmas 6.4-6.6 in Theorem 6.3, then we obtain the second main result of this paper. 
Theorem 6.7. Let $X$ and $Y$ be two non-crossing matchings with $d$ and e arches, respectively. Then, for $m \geq 3 d-e$,

$$
\begin{aligned}
& A_{X, Y}(m)=S S Y T(\lambda(X), m-2 d+e+1) \cdot S S Y T(\lambda(Y), m-d+1) \\
& +\sum_{\begin{array}{c}
\mathcal{E}_{1}, \mathcal{E}_{2}, \mathcal{F}_{1}, \mathcal{F}_{2} \\
\end{array}}\left(a_{X}\left(\mathcal{E}_{1}, \mathcal{E}_{2}\right) a_{Y}\left(\mathcal{F}_{1}, \mathcal{F}_{2}\right)\right. \\
& \quad r\left(\lambda\left(\mathcal{E}_{1}\right), \lambda\left(\mathcal{F}_{2}\right), d-1, e-1, m-3 d+e+2,2 d-2 e+1\right) \\
& \left.\quad \cdot r\left(\lambda\left(\mathcal{F}_{1}\right), \lambda\left(\mathcal{E}_{2}\right), e-1, d-1, m-d-e+2,1\right)\right),
\end{aligned}
$$

where the sum is taken over all subsets $\mathcal{E}_{1}, \mathcal{E}_{2}$ of $\{1,2, \ldots, 2 d-2\}$ and all subsets $\mathcal{F}_{1}, \mathcal{F}_{2}$ of $\{1,2, \ldots, 2 e-2\}$ such that $\lambda\left(\mathcal{E}_{1}\right) \varsubsetneqq \lambda(X), \lambda\left(\mathcal{E}_{2}\right) \subseteq \lambda(X)^{\prime}, \lambda\left(\mathcal{F}_{1}\right) \varsubsetneqq \lambda(Y)$, and $\lambda\left(\mathcal{F}_{2}\right) \subseteq$ $\lambda(Y)^{\prime}$.

Proof. In the sum on the right-hand side of (6.1) we single out the term where $\lambda\left(\mathcal{E}_{1}\right)=$ $\lambda(X), \lambda\left(\mathcal{E}_{2}\right)=\lambda\left(\mathcal{F}_{2}\right)=\emptyset$, and $\lambda\left(\mathcal{F}_{1}\right)=\lambda(Y)$. By Lemma 6.6, in that case we have $a_{X}\left(\mathcal{E}_{1}, \mathcal{E}_{2}\right)=a_{Y}\left(\mathcal{F}_{1}, \mathcal{F}_{2}\right)=1$, and by Lemma 6.5 we have

$$
r(\lambda(X), \emptyset, d-1, e-1, m-3 d+e+2,2 d-2 e+1)=r(\lambda(X), d-1, m-3 d+e+2)
$$

and

$$
r(\lambda(Y), \emptyset, e-1, d-1, m-d-e+2,1)=r(\lambda(Y), e-1, m-d-e+2),
$$

where $r(\lambda, d, h)$ is short for the number of rhombus tilings of the region $R(\lambda, d, h)$. By Theorem 2.2, the latter numbers are given by the corresponding specializations in formula (2.2). Thus, we obtain the first term on the right-hand side of (6.4). The asserted description of the summation range of the sum on the right-hand side of (6.4) follows then from Lemma 6.6.

Zuber's Conjecture 1.2, in the case that $m \geq 3 d-e$, is now a simple corollary of the above theorem.

Proof of Conjecture 1.2 for $m \geq 3 d-e$. The polynomiality in $m$ of $A_{X, Y}(m)$ is obvious from (6.1) and Lemma 6.4. The assertion about the integrality of the coefficients of the "numerator" polynomial $P_{X, Y}(m)$ follows as well from Lemma 6.4. Finally, to see that the leading coefficient of $P_{X, Y}(m)$ is $\operatorname{dim}(\lambda(X)) \cdot \operatorname{dim}(\lambda(Y))$, one first observes that the leading term in (6.4) appears in the first term on the right-hand side. The claim follows now by a combination of (2.2) and (2.1).

Remark. The reader may wonder why we are not able to prove Conjecture 1.2 for the "small" values of $m$, that is, for the range $0 \leq m<3 d-e$. While we could try to imitate the approach of Section 5, and place $X$ around the top-right corner and $Y$ around the bottom-left corner of the square grid $Q_{n}$, the problem arises when Lemma 3.1 is applied to determine the fixed edges. Unfortunately, here, it is not the case that every grid vertex is on at least one fixed edge. On the contrary, there will be a strip without any fixed edge extending between the places where $X$ and $Y$ sit. Hence, the rhombus tiling arguments of Section 5 do not apply. 


\section{Auxiliary Results for FPL COnfigurations in a triangle}

The purpose of this section is to provide the proofs of Lemmas 4.1, 6.2 and 6.6. These will be consequences of Theorem 7.1 below.

For convenience, in this section we will encode Ferrers diagrams $\lambda$ which come from non-crossing matchings $X$ or sets $\mathcal{E}$ in form of paths consisting of up-steps $(1,1)$ and down-steps $(1,-1)$, which start at the origin. To obtain the path corresponding to a Ferrers diagram $\lambda$ contained in the square $\left(d^{d}\right)$, we trace the lower-right contour of $\lambda$ (including the parts along the left side and the upper side of the square), and rotate it by $45^{\circ}$. See Figure 7.1 for an illustration in the case of $\lambda=(4,2,1)$ when viewed as a Ferrers diagram contained in $\left(5^{5}\right)$.
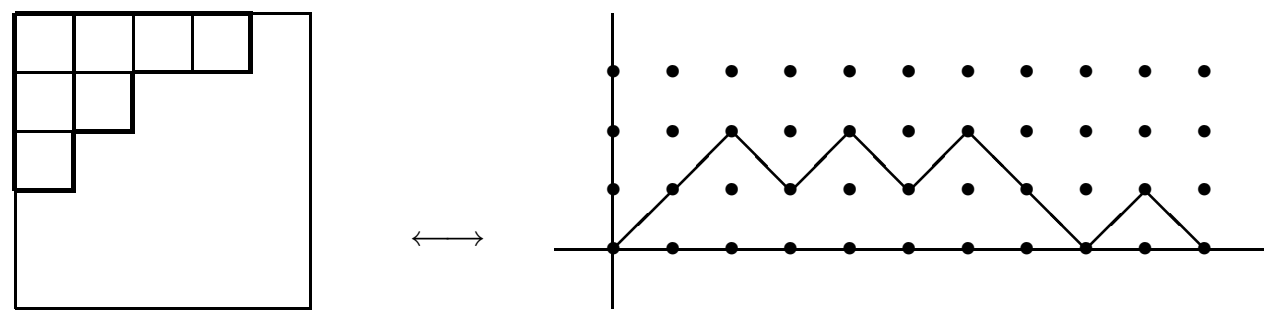

FIgURE 7.1. A Ferrers diagram embedded in a square and its corresponding path

Given a non-crossing matching $X$ with $d$ arches, we shall write $\pi(X)$ for the path corresponding to $\lambda(X)$ as contained in $\left(d^{d}\right)$. For example, for $X_{0}$ the non-crossing matching $\{1 \leftrightarrow 8,2 \leftrightarrow 3,4 \leftrightarrow 5,6 \leftrightarrow 7,9 \leftrightarrow 10\}$, the path $\pi\left(X_{0}\right)$ is the one in Figure 7.1. Clearly, $\pi(X)$ will always be a Dyck path, that is, a path which never passes below the $x$-axis and finally ends on the $x$-axis.

On the other hand, given a subset $\mathcal{E}$ of $\{1,2, \ldots, 2 d-2\}$, let $c_{\mathcal{E}}$ be the corresponding binary string as described at the beginning of Section 4 . We shall denote by $\pi(\mathcal{E})$ the path which arises from the string $0 c_{\mathcal{E}} 1$ (i.e., we prepended a 0 and appended a 1 to $c_{\mathcal{E}}$ ) by starting the path at the origin, and then reading $0 c_{\mathcal{E}} 1$ from left to right, interpreting a 0 as an up-step $(1,1)$, and a 1 as a down-step $(1,-1)$. For example, if $d=5$ and $\mathcal{E}_{0}=\{1,2,4,8\}$, then $c_{\mathcal{E}_{0}}=00101110$, and the path $\pi\left(\mathcal{E}_{0}\right)$ is the thin path on the right in Figure 7.2. It should be noted that, in general, $\pi(\mathcal{E})$ need not at all be a Dyck path; neither does it have to end on the $x$-axis, nor does it have to stay above the $x$-axis.

Now let us consider a triangular region $\mathcal{R}$ below a non-crossing matching $X$ with $d$ arches, as the region $A B K$ in Figures 4.1 or 6.2 . (The same arguments will apply to the region $F G M$ in Figure 6.2.) We also include the $2 \times(2 d-1)$ vertices on the left and the right. See the left part of Figure 7.2 for an example with $d=5$. As earlier, we denote the segment connecting the points half a unit to the left of $A$ and $K$ by $\xi_{1}$. In accordance with the situations which we face in Sections 4 and 6, the FPL configurations that we consider in this section will always contain all the horizontal edges along the left border of $\mathcal{R}$, that is, the horizontal edges crossing the segment $\xi_{1}$, and all the horizontal edges along the right border of $\mathcal{R}$. Moreover, we will only consider FPL configurations 

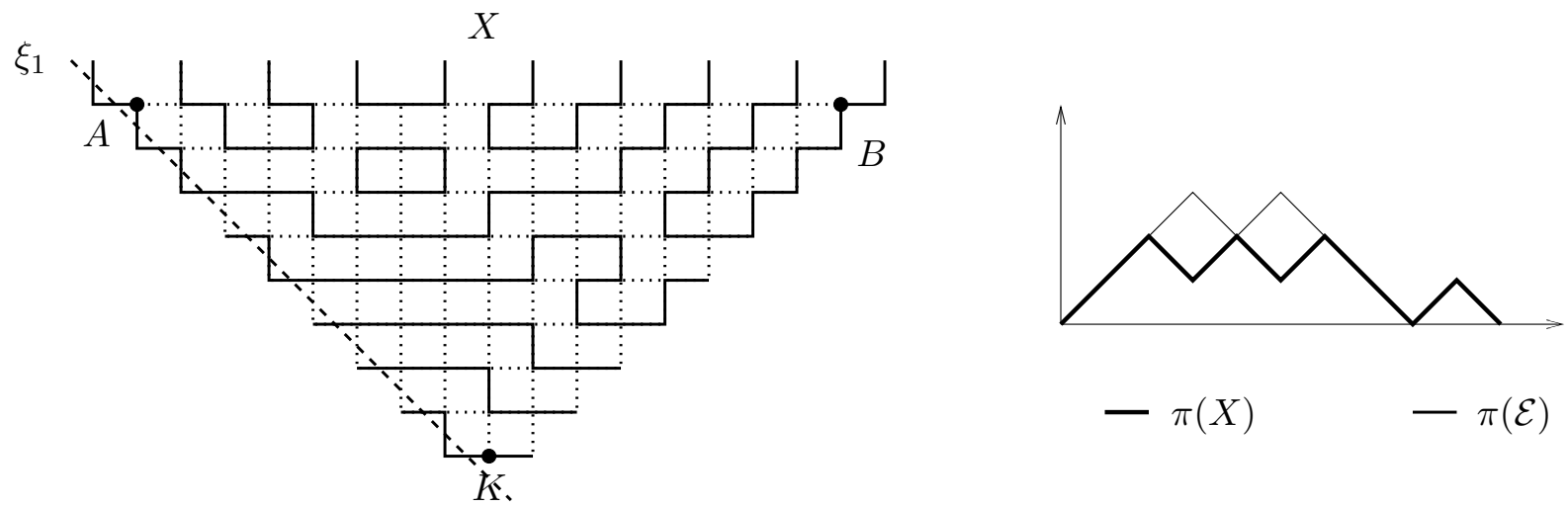

Figure 7.2. The triangular region $\mathcal{R}$ with an example of an FPL configuration, and the paths $\pi(\mathcal{E})$ and $\pi(X)$.

consisting exclusively of loops connecting external edges occupied by $X$ or loops which enter $\mathcal{R}$ from the left and exit $\mathcal{R}$ on the right. (The latter correspond to some of the $m$ "parallel" loops in our two problems.) Let us call these FPL configurations $\mathcal{R}-F P L$ configurations.

Given an $\mathcal{R}$-FPL configurations, we encode the set of vertical edges crossing $\xi_{1}$ which are taken by the configuration by a subset $\mathcal{E}$ of $\{1,2, \ldots, 2 d-2\}$, in the same way as we did before. (This is the same notation as in Section 4. In Section 6, this set was denoted by $\mathcal{E}_{1}$.) Figure 7.2 shows a possible $\mathcal{R}$-FPL configuration inside $\mathcal{R}$ in the case $d=5$. There, the induced matching is the matching $X_{0}$ from above, and the subset which encodes the vertical edges crossing $\xi_{1}$ which are occupied by the $\mathcal{R}$-FPL configuration is the set $\mathcal{E}_{0}$ from above. The right part of Figure 7.2 shows the corresponding paths $\pi\left(X_{0}\right)$ and $\pi\left(\mathcal{E}_{0}\right)$. It should be noted that we do not suppose that $|\mathcal{E}|=d-1$; this will be a consequence of the subsequent considerations.

Given a path $\pi($.$) (that is, either a path \pi(X)$ or a path $\pi(\mathcal{E})$ ), we let $\pi_{i}($.$) be the$ $y$-coordinate of the point on the path which has $x$-coordinate $i$. For example, we have $\pi_{2}\left(X_{0}\right)=1$ and $\pi_{2}\left(\mathcal{E}_{0}\right)=3$ (see Figure 7.2). Clearly, the two $y$-coordinates $\pi_{i}(\mathcal{E})$ and $\pi_{i}(X)$ differ by an even integer, so that we can define the integers

$$
h_{i}=\frac{\pi_{i}(\mathcal{E})-\pi_{i}(X)}{2}, \quad i=1,2, \ldots, 2 d-1
$$

In the sequel, for convenience, we shall in fact consider a rotated picture, see Figure 7.3 for the rotated version of the $\mathcal{R}$-FPL configuration in $\mathcal{R}$ from Figure 7.2. (The numbers on the right should be ignored at the moment.) We always also include the external edges occupied by the matching $X$.

We now cut the (rotated) region $\mathcal{R}$ in slices, as indicated in Figure 7.3. We call the cutting lines $\Lambda_{i}$ and $\mathcal{V}_{i}$, respectively, $i=1,2, \ldots, 2 d-1$. Given an $\mathcal{R}$-FPL configuration, we assign one of four types to every vertex on one of these lines, as we explain below. The reader is advised to look at Figure 7.4 while reading the definition of these types. 


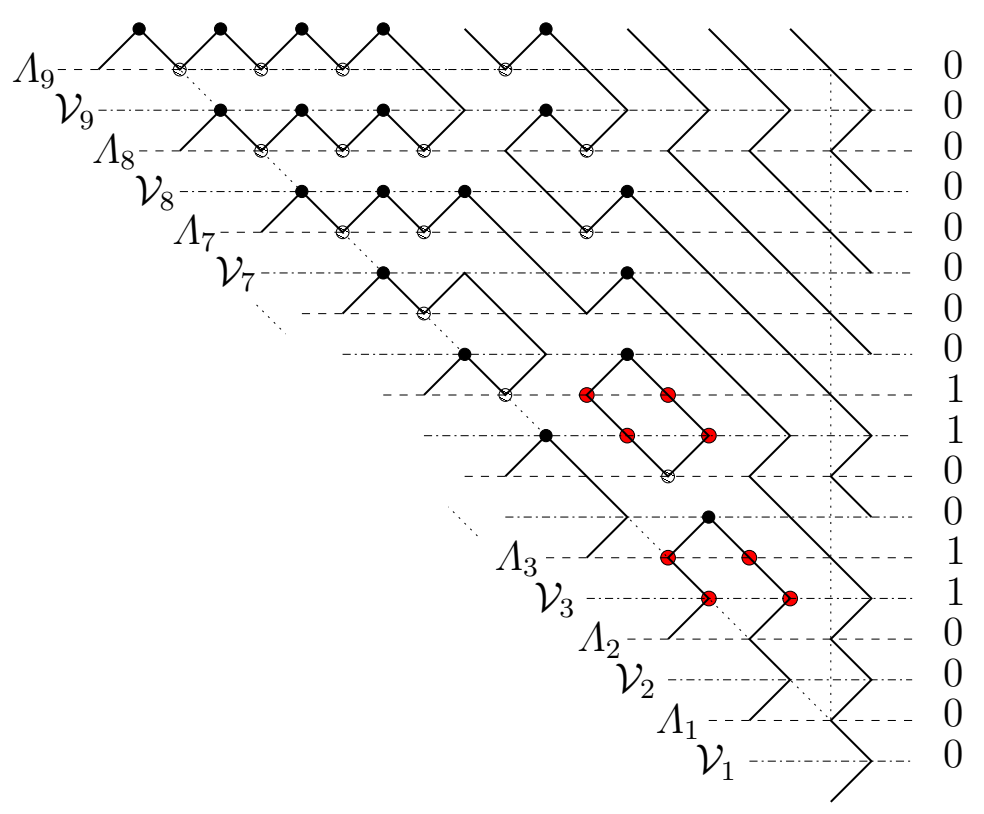

FiguRE 7.3. The region $\mathcal{R}$ after rotation

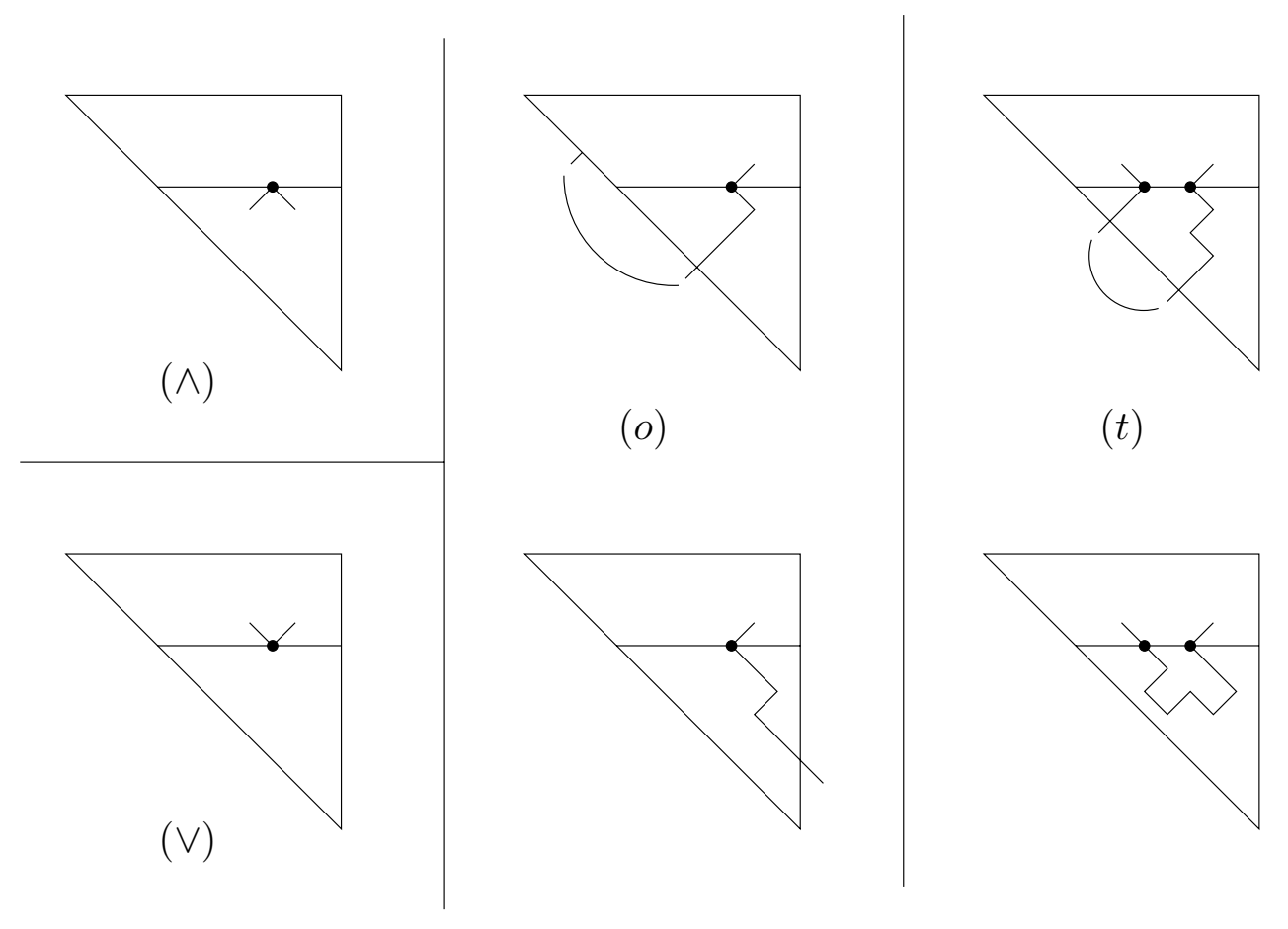

Figure 7.4. The four types of vertices

Let $v$ be a vertex on line $l$, where $l$ is either $\Lambda_{i}$ or $\mathcal{V}_{i}$, for some $i$. We say that $v$ has type $\vee$ (respectively $\wedge$ ) if the two edges attached to $v$ are both above (respectively below) $l$. 
If we are not in one of these cases, then there is exactly one edge $e$ attached to $v$ which is below $l$. The edge $e$ is on a loop of the configuration. Let us start with $e$ and follow the part of the loop below $l$. At some point, we shall either exit $\mathcal{R}$ or pass above $l$. Let us call the last edge before this happens $e^{\prime}$. The two remaining types of vertices are defined depending on the location of $e^{\prime}$ : if $e^{\prime}$ crosses $\xi_{1}$ (the right border of the rotated version of $\mathcal{R})$, or is an external edge linked, via $X$, to another external edge situated strictly above $l$, then we say that $v$ has type $o$. (Here, "o" stands for "out".) If $e^{\prime}$ is incident to $l$, or is an external edge linked, via $X$, to another external edge situated below $l$, then we say that $v$ has type $t$. See Figure 7.4. An important feature of vertices of type $t$ is that they always go in pairs. (This feature explains also the terminology " $t$ ", which stands for "twin".) By convention, the right-most vertex of $\mathcal{V}_{i}$ is of type $o$ if it is not linked to its neighbour on $\Lambda_{i-1}$.

Now we want to count the vertices on each line according to their type: let $\Lambda_{\vee}(i)$, $\Lambda_{\wedge}(i), \Lambda_{t}(i)$, and $\Lambda_{o}(i)$ be the respective numbers of vertices of $\Lambda_{i}$ of type $\vee, \wedge, t$, and $o$. Likewise we introduce the quantities $V_{\vee}(i), V_{\wedge}(i), V_{t}(i)$, and $V_{o}(i)$ for the line $\mathcal{V}_{i}$. Since vertices of type $t$ go in pairs, $\Lambda_{t}(i)$ and $V_{t}(i)$ are even numbers. We are now ready to state the result from which Lemmas 4.1, 6.2 and 6.6 will follow.

Theorem 7.1. If $1 \leq i \leq 2 d-1$, we have

$$
\Lambda_{\wedge}(i)+\frac{1}{2} \Lambda_{t}(i)=V_{\vee}(i)+\frac{1}{2} V_{t}(i)=h_{i} .
$$

For an illustration of this result, the reader should consult Figure 7.3. The numbers along the right rim of the figure are the quantities $\Lambda_{\wedge}(i)+\frac{1}{2} \Lambda_{t}(i)$ and $V_{\vee}(i)+\frac{1}{2} V_{t}(i)$. They are indeed equal to the numbers $h_{i}$, as can be seen by comparison with the right part of Figure 7.2.

Theorem 7.1 is an immediate corollary of the following two lemmas, as can be easily seen by an induction with respect to $i$. In the lemmas, we use the notations $\Delta^{(\Lambda-\mathcal{V})}(i)=$ $\left(\Lambda_{\wedge}(i)+\frac{1}{2} \Lambda_{t}(i)\right)-\left(V_{\vee}(i)+\frac{1}{2} V_{t}(i)\right)$ and $\Delta^{(\mathcal{V}-\Lambda)}(i)=\left(V_{\vee}(i)+\frac{1}{2} V_{t}(i)\right)-\left(\Lambda_{\wedge}(i-1)+\frac{1}{2} \Lambda_{t}(i-1)\right)$ for the differences of the quantities which appear in the theorem.

Lemma 7.2. For $i=1,2, \ldots, 2 d-1$, we have $\Delta^{(\Lambda-\mathcal{V})}(i)=0$.

Lemma 7.3. For $i=1,2, \ldots, 2 d-1$, we have

$$
\Delta^{(\mathcal{V}-\Lambda)}(i)=\left\{\begin{aligned}
1 & s_{i}(\mathcal{E}) \text { is an up-step and } s_{i}(X) \text { is a down-step } \\
-1 & s_{i}(\mathcal{E}) \text { is a down-step and } s_{i}(X) \text { is an up-step } \\
0 & \text { otherwise, }
\end{aligned}\right.
$$

where $s_{i}(\mathcal{E})$ denotes the $i$-th step of the path $\pi(\mathcal{E})$, with the analogous meaning for $s_{i}(X)$.

Proof of Lemmas 7.2 and 7.3. To begin with, we have to introduce some terminology and notation. We consider the slices between the lines $\mathcal{V}_{i}$ and $\Lambda_{i}$ on the one hand, $i=$ $1,2, \ldots, 2 d-1$, and between the lines $\Lambda_{i-1}$ and $\mathcal{V}_{i}$ on the other hand, $i=2,3, \ldots, 2 d-1$, separately. See Figure 7.5. One should note that the vertex on the very left of $\mathcal{V}_{i}$ is always incident to an external edge, while the right-most vertices of $\mathcal{V}_{i}$ and $\Lambda_{i}$ are connected by 
one of the horizontal edges (before rotation) crossing $\xi_{1}$. These edges are indicated as thick edges in Figure 7.5.

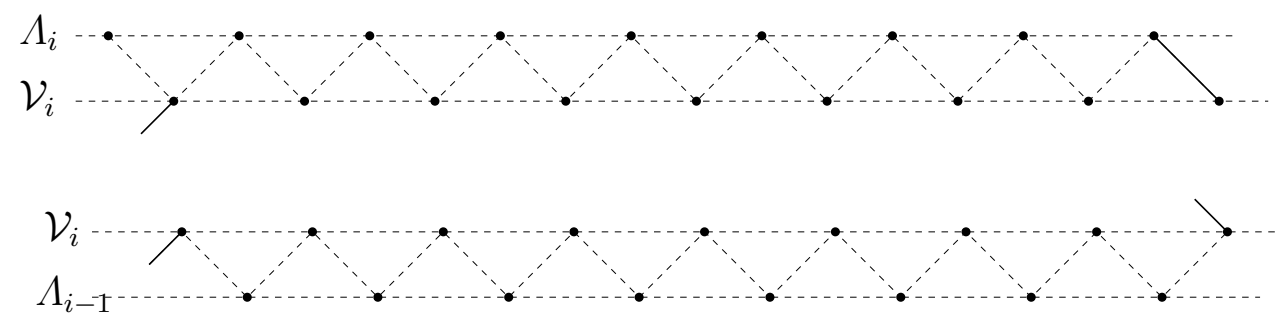

Figure 7.5. The two kinds of slices

The restriction of an $\mathcal{R}$-FPL configuration to such a slice is a graph. Let us call any connected component of such a graph which is not an isolated vertex simply component. Two components might be linked in the sense that each of them has a vertex such that the two vertices together form a pair of vertices of type $t$. (The two vertices may be on the same component, in which case this component is linked to itself). When taking the reflexive and transitive closure of this relation, we obtain an equivalence relation on the set of components. We call the equivalence classes of this relation groups. In other words, groups are minimal unions of components such that one can pass from one component to another by a series of links.

For every subset of vertices $E$ of the graph, let us introduce the notation $\Delta_{E}^{(\Lambda-\mathcal{V})}$ (respectively $\left.\Delta_{E}^{(\mathcal{V}-\Lambda)}\right)$ to represent the quantity $\Delta^{(\Lambda-\mathcal{V})}\left(\right.$ respectively $\left.\Delta^{(\mathcal{V}-\Lambda)}\right)$ where the count of vertices is restricted to those in $E$. So, for $g$ a group, $c$ a component, or for $I$ sol the set of isolated vertices, we have the numbers $\Delta_{g}^{(\Lambda-\mathcal{V})}, \Delta_{g}^{(\mathcal{V}-\Lambda)}, \Delta_{c}^{(\Lambda-\mathcal{V})}, \Delta_{c}^{(\mathcal{V}-\Lambda)}, \Delta_{\text {Isol }}^{(\Lambda-\mathcal{V})}$, and $\Delta_{\text {Isol }}^{(\mathcal{V}-\Lambda)}$. As all groups of a slice, together with the set of isolated vertices, form a partition of this slice, we have

$$
\Delta^{(\Lambda-\mathcal{V})}=\Delta_{\text {Isol }}^{(\Lambda-\mathcal{V})}+\sum_{g \text { a group }} \Delta_{g}^{(\Lambda-\mathcal{V})} \text { and } \quad \Delta^{(\mathcal{V}-\Lambda)}=\Delta_{\text {Isol }}^{(\mathcal{V}-\Lambda)}+\sum_{g \text { a group }} \Delta_{g}^{(\mathcal{V}-\Lambda)}
$$

In the sequel, when making pictures, we allow ourselves the following simplifications. First, we write two linked components of a given group next to each other even if they may be in a different order in an actual configuration; some of the components may have to be replaced by their left-right symmetric image in the process. This is allowed since this does not change the quantities $\Delta_{g}$. Second, we write any component $c$ in the reduced way indicated in the column "Simplification" in Figure 7.6. Again, this is allowed since this does not change the value of the corresponding $\Delta_{c}$. An actual group and its simplified version are shown in Figure 7.7.

We are now ready for the proof of Lemma 7.2. First, we claim that we have $\Delta_{\text {Isol }}^{(\Lambda-\mathcal{V})}=0$, where $I$ sol denotes the set of isolated vertices in the slice between $\mathcal{V}_{i}$ and $\Lambda_{i}$. Indeed, the isolated vertices in this slice are of type $\wedge$ (respectively $\vee$ ) if they belong to $\mathcal{V}_{i}$ (respectively $\left.\Lambda_{i}\right)$. Thus, they do not contribute to $\Delta_{\text {Isol }}^{(\Lambda-\mathcal{V})}$, which is therefore 0 . Next we have to look 


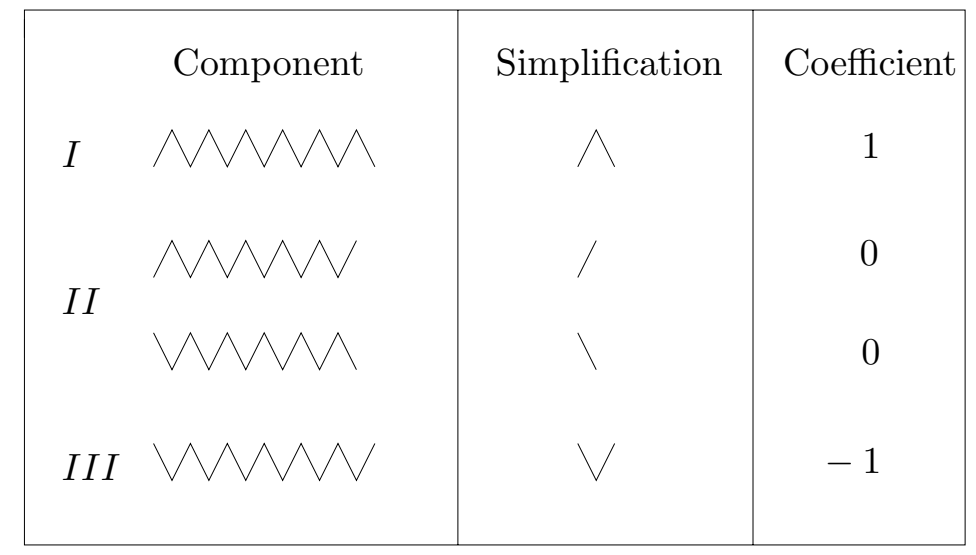

FiguRE 7.6. The possible components, their simplified version, and the coefficients used in the proof of Lemma 7.3

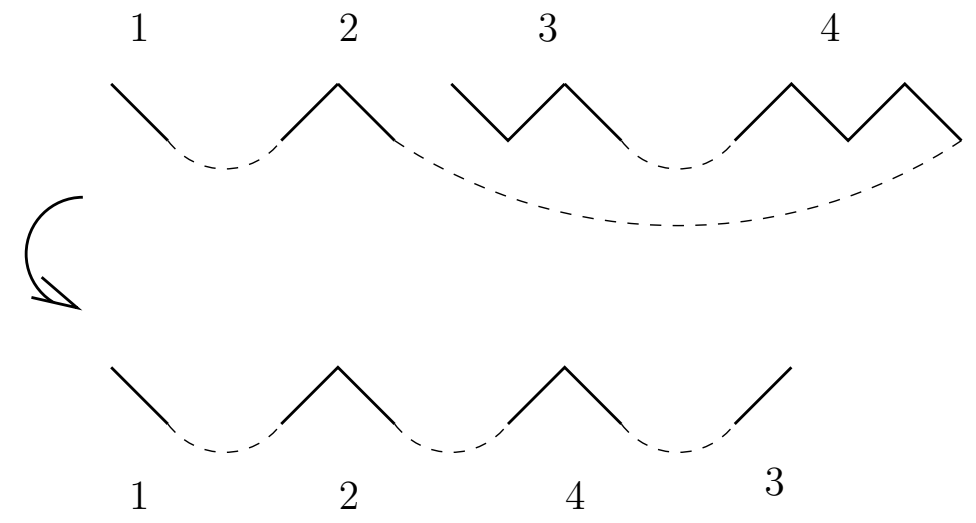

FiguRE 7.7. A group (top) and its simplified version (bottom)

closer at groups in order to evaluate $\Delta^{(\Lambda-\mathcal{V})}(i)$. The different possibilities are illustrated in Figure 7.8. It is easy to see that these are the only possible groups here, and that for each of them we have $\Delta_{g}^{(\Lambda-\mathcal{V})}=0$. Lemma 7.2 is thus proved.

Finally, we turn to the proof of Lemma 7.3, which is more difficult, because for the isolated vertices $I s o l$ in the slice between $\Lambda_{i-1}$ and $\mathcal{V}_{i}$ we do not have $\Delta_{\text {Isol }}^{(\mathcal{V}-\Lambda)}=0$ in general, and because there are more groups to consider. First we use a trick that allows us to get rid of $\Delta_{I s o l}^{(\mathcal{V}-\Lambda)}$ by suitably modifying the $\Delta_{g}^{(\mathcal{V}-\Lambda)}$ 's. Let us consider the slice between $\Lambda_{i-1}$ and $\mathcal{V}_{i}$. It alternates between components and blocks of isolated vertices. (When we say "alternate," then we also allow for empty blocks of isolated vertices.) A block $b$ of isolated vertices is generally surrounded by two components. Notice that $\Delta_{b}^{(\mathcal{V}-\Lambda)}$ is equal to 1 (respectively -1 , respectively 0 ) if the left-most vertex and the right-most vertex of $b$ are both on $\mathcal{V}_{i}$ (respectively both on $\Lambda_{i-1}$, respectively one on each line). We may split these values as $1=\frac{1}{2}+\frac{1}{2},-1=\left(-\frac{1}{2}\right)+\left(-\frac{1}{2}\right)$, and $0=\frac{1}{2}+\left(-\frac{1}{2}\right)$, respectively $0=\left(-\frac{1}{2}\right)+\frac{1}{2}$, and the idea is to "move" the left summand to the component to the left 

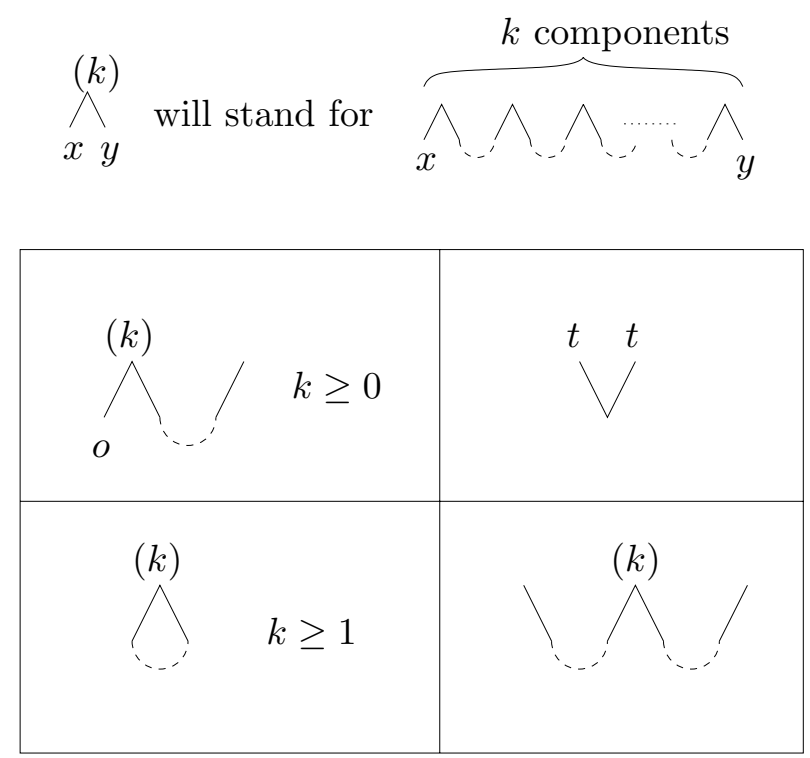

FIGURE 7.8. All possible groups for Lemma 7.2

and the right summand to the component to the right of $b$. Thus, every component $c$ will accumulate one or two values out of $\left\{\frac{1}{2},-\frac{1}{2}\right\}$. If the component $c$ is in between two blocks of isolated vertices (recall: these blocks may also be empty blocks), then the sum of these two values is 1, 0, or -1 . Column "Coefficient" in Figure 7.6 shows this sum for the various types of components. Thus, for such a component, we put

$$
\Delta_{c}^{\prime}=\Delta_{c}^{(\mathcal{V}-\Lambda)}+\varepsilon,
$$

where $\varepsilon \in\{-1,0,1\}$ as indicated in the column "Coefficient" in Figure 7.6. If a component $c$ is to the very left of the slice, then there is only a block of isolated vertices to the right. In that case we also make the definition (7.1), but with $\varepsilon=\frac{1}{2}$ if the right-most vertex of $c$ is on $\Lambda_{i-1}$, and with $\varepsilon=-\frac{1}{2}$ if the right-most vertex of $c$ is on $\mathcal{V}_{i}$. Similarly, if a component $c$ is to the very right of the slice, then there is only a block of isolated vertices to the left. In that case we also make the definition (7.1), but with $\varepsilon=\frac{1}{2}$ if the left-most vertex of $c$ is on $\Lambda_{i-1}$, and with $\varepsilon=-\frac{1}{2}$ if the left-most vertex of $c$ is on $\mathcal{V}_{i}$. See Figure 7.9 for these latter components. (There, $s_{i}(\mathcal{E})$ and $s_{i}(X)$ are the $i$-th steps of the paths $\pi(\mathcal{E})$ and $\pi(X)$, respectively, and "up" is short for "up-step,", while "down" is short for "down-step.") Finally, we put $\Delta_{g}^{\prime}=\sum \Delta_{c}^{\prime}$. Then we have

$$
\Delta^{(\mathcal{V}-\Lambda)}=\sum_{g \text { a group }} \Delta_{g}^{\prime}
$$

Now for each group in Figure 7.8 (that is, the groups consisting exclusively of components between two blocks of isolated vertices), one verifies that $\Delta_{g}^{\prime}=0$. All possible cases for the remaining groups (that is, groups with components to the very right or to the very left of the slice) are dealt with in Figure 7.9. There, we use the simplifications 


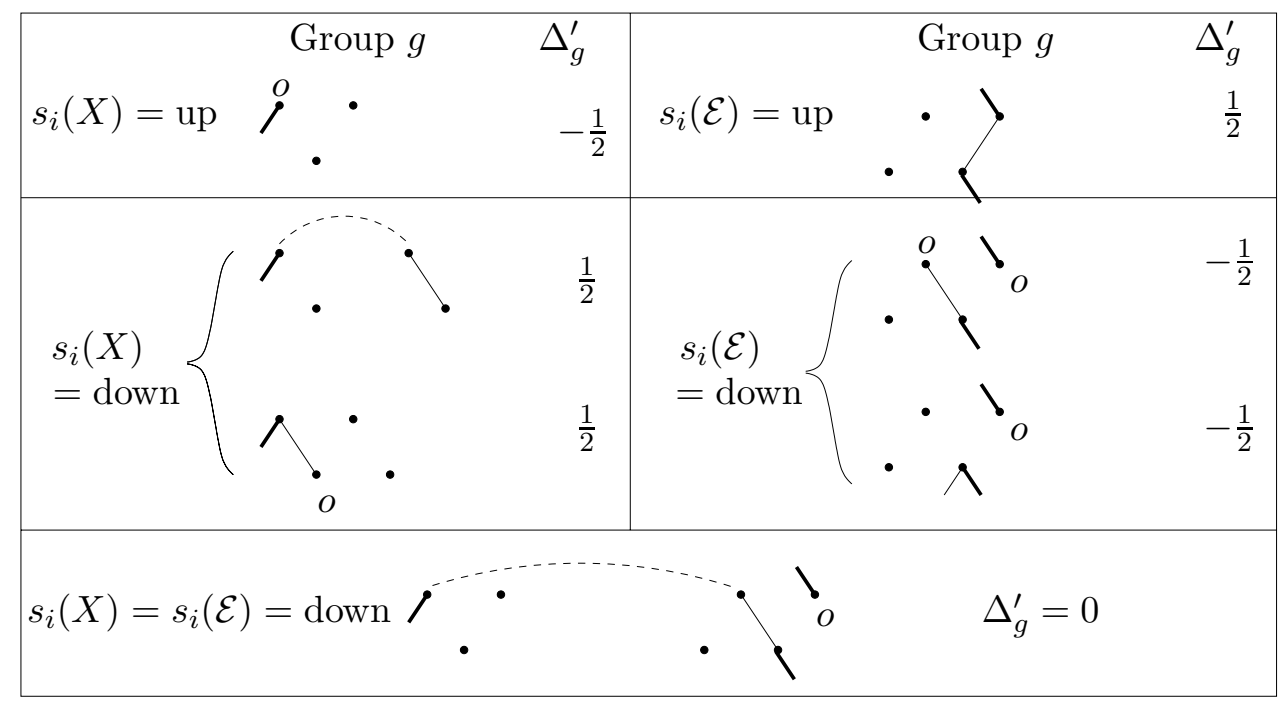

FiguRE 7.9. "Side" groups

mentioned above plus an additional one: the pairs of vertices of type $t$ on the line $\Lambda_{i-1}$ are not represented because they do not modify the quantities $\Delta_{g}$. It is not difficult to verify that, when the corresponding values of $\Delta_{g}^{\prime}$ given in Figure 7.9 are substituted in (7.2), the assertion of Lemma 7.3 follows.

Corollary 7.4. (1) If there exists an $\mathcal{R}-F P L$ configuration, then the path $\pi(\mathcal{E})$ is always weakly above the path $\pi(X)$. Equivalently, $\lambda(\mathcal{E}) \subseteq \lambda(X)$.

(2) If there exists an $\mathcal{R}-F P L$ configuration, then the path $\pi(\mathcal{E})$ is a Dyck path. In particular, $\mathcal{E}$ has exactly $d-1$ elements.

(3) If $\pi(\mathcal{E})=\pi(X)$ (or, equivalently, $\lambda(\mathcal{E})=\lambda(X)$ ), then there is exactly one $\mathcal{R}-F P L$ configuration filling the region $\mathcal{R}$.

Proof. The assertion (1) is just a reformulation of $h_{i} \geq 0$, which is a direct consequence of Theorem 7.1 .

For proving (2), we first observe that, because of (1), and because we know that $\pi(X)$ is a Dyck path, $\pi(\mathcal{E})$ is certainly a path which never passes below the $x$-axis. Thus, since the last steps of $\pi(X)$ and $\pi(\mathcal{E})$ are down-steps by definition, it suffices to prove that $h_{2 d-1}=0$. To see the latter, we look at the line $\Lambda_{2 d-1}$. All its vertices are incident to one of the horizontal edges along the right border of $\mathcal{R}$ (the terminology refers to the situation before rotation). Thus, none of these vertices can be of type $\wedge$. Furthermore, since our $\mathcal{R}$-FPL configurations contain either loops connecting external edges occupied by $X$ or enter $\mathcal{R}$ from left and exit on the right, none of the vertices on $\Lambda_{2 d-1}$ can be of type $t$. Thus, by Theorem 7.1 , we have $h_{2 d-1}=\Lambda_{\wedge}(i)+\frac{1}{2} \Lambda_{t}(i)=0$, as desired.

Finally, we prove assertion $(3)$. Let $\pi(\mathcal{E})=\pi(X)$. In this case we have $h_{i}=0$ for all $i$. Thus, according to Theorem 7.1, the task is to construct $\mathcal{R}$-FPL configurations such that the lines $\Lambda_{i}$ contain exclusively vertices of types $\vee$ and $o$, while lines $\mathcal{V}_{i}$ contain exclusively 
vertices of types $\wedge$ and $o$. We claim that, if one proceeds slice by slice, beginning from the bottom (that is, the slice between $\mathcal{V}_{1}$ and $\Lambda_{1}$ ), and working upwards, then there is a unique such configuration.

To see this, the crucial observation is that, since vertices of type $t$ are forbidden, there cannot be any closed loops in $\mathcal{R}$ or loops which start and end on the top-most line $\Lambda_{2 d-1}$. Thus, inductively, if we have already worked our way up to line $\mathcal{V}_{i}$, then, on $\mathcal{V}_{i}$, the vertices of type $\wedge$ will be on the left and the vertices of type $o$ will be on the right. (It is also possible that there are no vertices of one of the two types.) See Figure 7.10. There, we have marked the external edge incident to the left-most vertex on $\mathcal{V}_{i}$ and the horizontal edge (before rotation) crossing $\xi_{1}$ which connects the right-most vertices of $\mathcal{V}_{i}$ and $\Lambda_{i}$ by thick lines. The latter edge forces us to connect the vertices of type $o$ on $\mathcal{V}_{i}$ diagonally up-left to the neighbouring vertices of $\Lambda_{i}$. Since the latter cannot be of type $\wedge$, they must be of type $o$. The remaining vertices on $\Lambda_{i}$ (which are all on the left) must be of type $\vee$.

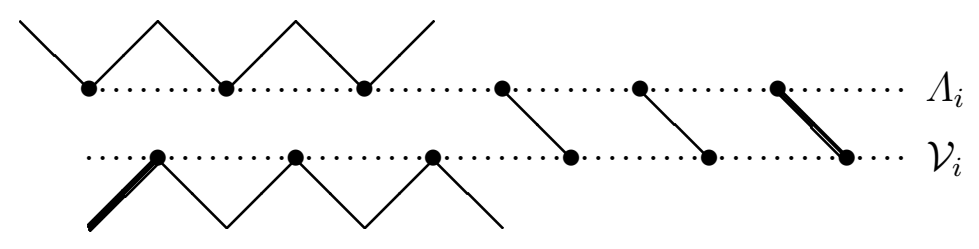

Figure 7.10. The unique filling of the slice between $\mathcal{V}_{i}$ and $\Lambda_{i}$

On the other hand, again inductively, if we have already worked our way up to line $\Lambda_{i-1}$, then, on $\Lambda_{i-1}$, the vertices of type $\vee$ will be on the left and the vertices of type $o$ will be on the right. (It is also possible that there are no vertices of one of the two types.) See Figures 7.11 and 7.12. There, we have marked the external edge incident to the left-most vertex on $\mathcal{V}_{i}$ and the horizontal edges (before rotation) crossing $\xi_{1}$ which are incident to the right-most vertices of $\Lambda_{i-1}$ and $\mathcal{V}_{i}$ by thick lines. Now there are two cases. Either the vertical edge (before rotation) connecting the right-most vertices of $\Lambda_{i-1}$ and $\mathcal{V}_{i}$ is among the vertical edges chosen via $\mathcal{E}$ or not. In the first case (cf. Figure 7.11), this edge forces us to connect the vertices of type $o$ and as well the right-most vertex of type $\vee$ on $\Lambda_{i-1}$ diagonally up-right to the neighbouring vertices of $\mathcal{V}_{i}$. Since the latter vertices cannot be of type $\vee$, they must be of type $o$. The remaining vertices on $\mathcal{V}_{i}$ (which are all on the left) must be of type $\wedge$. In the second case (cf. Figure 7.11), the missing edge on the very right forces us to connect the vertices of type $o$ on $\Lambda_{i-1}$ diagonally up-left to the neighbouring vertices of $\mathcal{V}_{i}$. Since the latter vertices cannot be of type $\vee$, they must be of type $o$, except for the left-most of those which is of type $\wedge$. The remaining vertices on $\mathcal{V}_{i}$ (which are all on the left) must also be of type $\wedge$.

This completes the proof of the corollary.

\section{REFERENCES}

[1] M. T. Batchelor, H. W. J. Blöte, B. Nienhuis and C. M. Yung, "Critical behaviour of the fully packed loop model on the square lattice," J. Phys. A 29 (1996), L399-L404. 


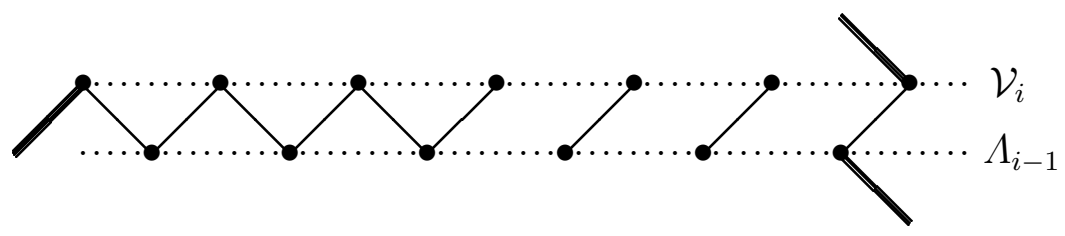

Figure 7.11. The unique filling of the slice between $\Lambda_{i-1}$ and $\mathcal{V}_{i}$ in Case 1

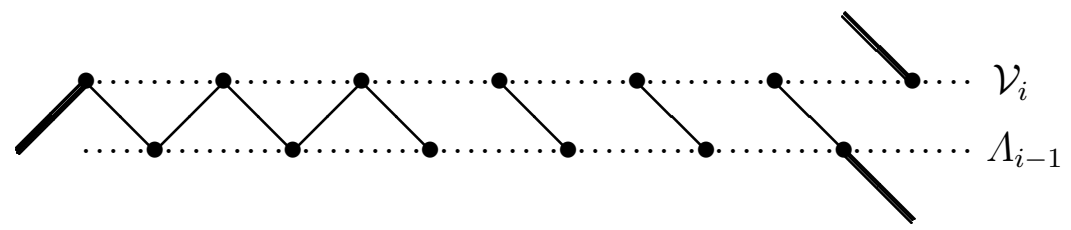

Figure 7.12. The unique filling of the slice between $\Lambda_{i-1}$ and $\mathcal{V}_{i}$ in Case 2

[2] F. Caselli and C. Krattenthaler, "Proof of two conjectures of Zuber on fully packed loop configurations," J. Combin. Theory Ser. A 108 (2004), 123-146.

[3] M. Ciucu, T. Eisenkölbl, C. Krattenthaler and D. Zare, "Enumeration of lozenge tilings of hexagons with a central triangular hole," J. Combin. Theory Ser. A 95 (2001), 251-334.

[4] M. Ciucu and C. Krattenthaler, "Plane partitions II: $5 \frac{1}{2}$ symmetry classes," Combinatorial Methods in Representation Theory, M. Kashiwara, K. Koike, S. Okada, I. Terada, H. Yamada, eds., Advanced Studies in Pure Mathematics, vol. 28, RIMS, Kyoto, 2000, 83-103.

[5] M. Ciucu and C. Krattenthaler, "Enumeration of lozenge tilings of hexagons with cut-off corners," J. Combin. Theory Ser. A 100 (2002), 201-231.

[6] J. de Gier, "Loops, matchings and alternating-sign matrices," Discr. Math., to appear. math. CO/0211285.

[7] P. Di Francesco, P. Zinn-Justin and J.-B. Zuber, "A bijection between classes of fully packed loops and plane partitions," Electron. J. Combin. 11 (1) (2004), Article \#R64, 11 pages.

[8] P. Di Francesco, P. Zinn-Justin and J.-B. Zuber, "Determinant formulae for some tiling problems and application to fully packed loops," Proceedings of the International Conference on Integrable Systems, Poitiers, June 2004, to appear. math-ph/0410002.

[9] P. Di Francesco and J.-B. Zuber, "On FPL configurations with four sets of nested arches," preprint. cond-mat/0403268.

[10] J. S. Frame, G. B. Robinson and R. M. Thrall, "The hook graphs of the symmetric group," Canad. J. Math. 6 (1954), 316-325.

[11] W. Fulton and J. Harris, "Representation Theory," Springer-Verlag, New York, 1991.

[12] I. M. Gessel and X. Viennot, "Binomial determinants, paths, and hook length formulae," Adv. in Math. 58 (1985), 300-321.

[13] I. M. Gessel and X. Viennot, Determinant, paths and plane partitions, preprint, 1989; available at http://www.cs.brandeis.edu/ ${ }^{\sim}$ ira.

[14] S. Karlin and J. L. McGregor, "Coincidence properties of birth-and-death processes," Pacific J. Math. 9 (1959), 1109-1140.

[15] S. Karlin and J. L. McGregor, "Coincidence probabilities," Pacific J. Math. 9 (1959), 1141-1164.

[16] B. Lindström, "On the vector representations of induced matroids," Bull. London Math. Soc. 5 (1973), 85-90. 
[17] S. Mitra, B. Nienhuis, J. de Gier and M. T. Batchelor, "Exact expression for correlations in the groundstate of the dense $O(1)$ model," Journal Statist. Mechanics: Theory and Experiment (2004), P09010. cond-mat/0401245.

[18] A.V. Razumov and Yu. G. Stroganov, "Combinatorial nature of the ground state vector of the $O(1)$ loop model," Theoret. Math. Phys. 138 (2004), 333-337. math.C0/0104216.

[19] R. P. Stanley, "Theory and applications of plane partitions: Part 2," Stud. Appl. Math. 50 (1971), 259-279.

[20] R. P. Stanley, "Enumerative Combinatorics," Vol. 2, Cambridge University Press, Cambridge, 1999.

[21] B. Wieland, "A large dihedral symmetry of the set of alternating sign matrices," Electronic J. Combin. 7 (2000), Article \#R37.

[22] J. B. Zuber, "On the counting of fully packed loops configurations. Some new conjectures," Electronic J. Combin. 11(1) (2004), Article \#R13.

*Institut Camille Jordan, Université Claude Bernard Lyon-I, 21, avenue Claude Bernard, F-69622 Villeurbanne Cedex, France

†Laboratoire de Recherche en Informatique, Université Paris-Sud, 91405 Orsay Cedex, FRANCE 